\title{
EQUIVARIANT COMPLETELY BOUNDED OPERATORS
}

\author{
Iain Raeburn, Allan M. Sinclair and Dana P. Williams
}

\begin{abstract}
An equivariant completely bounded linear operator between two $C^{*}$-algebras acted on by an amenable group is shown to lift to a completely bounded operator between the crossed products that is equivariant with respect to the dual coactions. A similar result is proved for coactions and dual actions. It is shown that the only equivariant linear operators that lift twice through the action and dual coaction of an infinite group are the completely bounded ones.
\end{abstract}

1. Introduction. Let $\Phi: A \rightarrow B$ be a bounded linear map between $C^{*}$-algebras, and let $\alpha: G \rightarrow$ Aut $A$ and $\beta: G \rightarrow$ Aut $B$ be actions of an amenable locally compact group $G$. If $\Phi$ is a homomorphism and is equivariant-i.e., $\Phi\left(\alpha_{s}(a)\right)=\beta_{s}(\Phi(a))$ for $s \in G, a \in A$-then it extends to a homomorphism $\Phi \times i$ from the crossed product $A \rtimes_{\alpha} G$ to $B \rtimes_{\beta} G$. On the other hand, if $C$ is another $C^{*}$-algebra, then for any completely bounded map $\Phi: A \rightarrow B$ there is a bounded operator $\Phi \times i: A \otimes C \rightarrow B \otimes C$; indeed, by taking $C$ to be the algebra $\mathscr{K}$ of compact operators, we can see that the complete boundedness of $\Phi$ is necessary for this to be true. We shall combine these results to prove that any equivariant completely bounded operator extends to a bounded map on the crossed product, formulate and prove the analogous results for crossed products by coactions of nonabelian groups, and investigate the extent to which complete boundedness is a necessary hypothesis.

We shall take similar approaches to the problems of lifting through actions and coactions. First, we prove equivariant versions of Stinespring's theorem [23] on completely positive maps into $\mathscr{B}(\mathscr{H})$, and we then show how to modify Wittstock's theorem [25] to write an equivariant symmetric completely bounded map into $\mathscr{B}(\mathscr{H})$ as a difference of equivariant completely positive ones. From these we can use standard symmetrisation techniques to deduce that an equivariant completely bounded operator $\Phi: A \rightarrow \mathscr{B}(\mathscr{H})$ can be realized in the form $\Phi(a)=T \pi(a) V$, where $\pi$ is part of a covariant representation $(\pi, U)$ of $(A, G, \alpha)$ in some larger Hilbert space. We can then define $\Phi \times i: A \rtimes_{\alpha} G \rightarrow \mathscr{B}(\mathscr{H})$ by the formula $\Phi \times i(z)=T(\pi \times U(z)) V$, and the result for $\Phi: A \rightarrow B$ follows easily. 
In the case of actions the extension of Stinespring's theorem is quite straightforward, and in fact has already been established by Paulsen [17] for other purposes. Our version of Wittstock's theorem is obtained from [25] by averaging using an invariant mean on the group $G$; of course, this means (sorry) we have to assume $G$ is amenable. It is possible that this hypothesis is not necessary at this stage (see Remark 3.4) but we shall also need it later because it plays a vital role in the present theory of crossed products by coactions. Because this theory is so far relatively undeveloped, the equivariant versions of Stinespring's and Wittstock's theorems for coactions are technically considerably harder. However, we believe that some of our constructions here could be of independent interest. For example, the Stinespring theorem includes as a special case a version of the GelfandNaimark-Segal construction of covariant representations from an invariant state, and this appears to involve non-trivial modifications of the usual construction (see the remark preceding Theorem 4.4). Our proof of Wittstock's theorem depends on an averaging construction using an "invariant mean" on the von Neumann algebra of a group and slice maps by non-normal functionals: similar methods have been used by Enock and Schwartz in their study of Kac algebras [6].

It is possible that a bounded lifting $\Phi \times i$ can exist even if the map $\Phi$ is not completely bounded. For example, suppose $G$ is abelian and acts trivially on both $A$ and $B$. Then

$$
A \rtimes_{\alpha} G \cong A \otimes C^{*}(G) \cong A \otimes C_{0}(\hat{G}) \cong C_{0}(\hat{G}, A),
$$

and any bounded linear map $\Phi: A \rightarrow B$ extends to a bounded map $\Phi \times i: A \rtimes_{\alpha} G \rightarrow B \rtimes_{\beta} G$, which is equivariant for the dual actions of $\hat{G}$. However, $\Phi \times i$ cannot then extend to a bounded map on the second crossed product unless $\Phi$ is completely bounded. The idea of the proof of this is simple: by Takai duality, $\left(A \rtimes_{\alpha} G\right) \rtimes_{\hat{\alpha}} \hat{G} \cong A \otimes \mathscr{K}$, and the existence of a bounded extension $(\Phi \times i) \times i$ would imply that $\Phi \otimes i: A \odot \mathscr{H} \rightarrow B \odot \mathscr{K}$ was bounded, so that $\Phi$ was completely bounded. In fact, we shall prove a version of this result for arbitrary amenable groups using non-abelian duality.

We have organized our work as follows. In $\S 2$, we set up our notation and develop the necessary background material on completely bounded operators, slice maps and crossed products. In particular, we discuss covariant representations and crossed products for coactions, and what we mean by saying a linear map is equivariant with respect to coactions. Section 3 contains our results on lifting equivariant maps to 
crossed products by actions, and $\S 4$ the analogous results for coactions. We have taken care to follow the same general pattern in these two sections. In $\S 5$ we prove that an equivariant map $\Phi:(A, G, \alpha) \rightarrow(B, G, \beta)$ lifts to the second crossed product $\left(A \rtimes_{\hat{\alpha}} G\right) \rtimes_{\hat{\alpha}} G$ if and only if $\Phi$ is completely bounded, and briefly discuss the analogous result for the case where $G$ coacts on $A$. Our last section contains an alternative treatment of the material in $\S 3$ for normal completely bounded maps on von Neumann algebras, and a brief discussion of how our results can be adapted for multilinear completely bounded operators.

\section{Preliminaries.}

Notation. We denote by $\otimes$ both the Hilbert space tensor product and the spatial (or minimal) tensor product of two $C^{*}$-algebras. If $\phi$ and $\psi$ are bounded (linear) operators on spaces $X$ and $Y$, then $\phi \otimes \psi$ will denote both the algebraically defined operator on the algebraic tensor product $X \odot Y$, and its extension to any completion-provided, of course, such an extension exists. The algebra of all bounded operators on a Hilbert space $\mathscr{H}$ will be denoted by $\mathscr{B}(\mathscr{H})$, and the ideal of compact operators by $\mathscr{K}(\mathscr{H})$ or just $\mathscr{K}$. If $A$ is a $C^{*}$-algebra, $M(A)$ will be its multiplier algebra ([2], [21, §3.12]). As well as the norm topology, $M(A)$ carries the strict topology, which is defined by the seminorms $x \rightarrow\|x a\|+\|a x\|$ for $a \in A$; note that $A$ is strictly dense in $M(A)$ [2]. We write 1 for the identity element of $A$ or $M(A)$, and $i$ for the identity map on $A$ or $M(A)$.

Completely bounded operators. Let $M_{n}(A)$ denote the $C^{*}$-algebra of $n \times n$ matrices with entries in a $C^{*}$-algebra $A$, which we shall identify with $A \otimes M_{n}(\mathbf{C})$. Let $\Phi: A \rightarrow B$ be a (linear) operator between $C^{*}$ algebras, and let $\Phi_{n}$ denote the tensor product $\Phi \otimes i: A \otimes M_{n} \rightarrow B \otimes M_{n}$; under the matrix identification, $\boldsymbol{\Phi}_{n}\left(\left(a_{i j}\right)\right)=\left(\Phi\left(a_{i j}\right)\right)$. Recall that $\boldsymbol{\Phi}$ is completely positive if $\Phi_{n} \geq 0$ for all $n$, is completely bounded if $\|\Phi\|_{c b}=\sup \left\{\left\|\Phi_{n}\right\|: n \in \mathbf{N}\right\}<\infty$, and is symmetric if $\Phi\left(a^{*}\right)=\Phi(a)^{*}$ for $a \in A$.

Wittstock's decomposition of a symmetric completely bounded operator from a $C^{*}$-algebra into $\mathscr{B}(\mathscr{H})$ (see Theorem 2.2 below) and the Stinespring representation theorem enable one to write each completely bounded operator $\Phi: A \rightarrow \mathscr{B}(\mathscr{H})$ in the form $\Phi(a)=T \pi(a) V$, where $\pi$ is a non-degenerate representation of $A$ on a Hilbert space $\mathscr{H}_{\pi}$ and $V: \mathscr{H} \rightarrow \mathscr{H}_{\pi}, T: \mathscr{H}_{\pi} \rightarrow \mathscr{H}$ are bounded operators such that $\|\Phi\|_{c b}=\|T\|\|V\|[19],[26]$; conversely, each such operator is completely bounded with $\|\phi\|_{c b} \leq\|T\|\|V\|$. This result immediately implies that a completely bounded operator $\Phi: A \rightarrow \mathscr{B}(\mathscr{H})$ extends to 
the enveloping von Neumann algebra $A^{* *}$, and hence to the multiplier algebra $M(A)$. More precisely, we have the following lemma.

LeMMA 2.1. Let $\Phi: A \rightarrow \mathscr{B}(\mathscr{H})$ be a completely bounded operator. Then there is a unique strict-to-*-strong continuous, completely bounded operator $\bar{\Phi}: M(A) \rightarrow \mathscr{B}(\mathscr{H})$ which extends $\Phi$. Further, $\|\bar{\Phi}\|_{c b}=\|\Phi\|_{c b}$, and $\bar{\Phi}$ is completely positive (symmetric) if and only if $\Phi$ is completely positive (symmetric).

Proof. Choose $T, V, \pi$ as above. Since $\pi$ is non-degenerate it extends to a strict-to-*-strong continuous representation $\bar{\pi}$ of $M(A)$ on $\mathscr{H}_{\pi}$. We set $\Phi(m)=T \bar{\pi}(m) V$ for $m \in M(A)$. Then $\bar{\Phi}$ extends $\Phi$, and is completely bounded with

$$
\|\Phi\|_{c b} \leq\|\bar{\Phi}\|_{c b} \leq\|T\|\|V\|=\|\Phi\|_{c b} .
$$

The uniqueness of $\bar{\Phi}$ follows from the strict density of $A$ in $M(A)$. If $\Phi$ is completely positive we can take $T=V^{*}$, and then $\bar{\Phi}$ is also completely positive since all maps of the form $m \mapsto V^{*} \rho(m) V$ are. An application of Wittstock's theorem gives the observation about symmetry of $\bar{\Phi}$. This proves the lemma.

When we use extensions of homomorphisms and completely bounded maps to operator algebras, we shall always be using the canonical strictly continuous extension given by this lemma-and we shall denote the extension by the same symbol. With this convention, we can state Wittstock's theorem ([25], [18], [19]) as follows.

THEOREM 2.2. Let $\Phi: A \rightarrow \mathscr{B}(\mathscr{H})$ be a symmetric completely bounded operator. Then there are completely positive operators $\Psi_{j}: A \rightarrow$ $\mathscr{B}(\mathscr{H})$ such that $\Phi=\Psi_{1}-\Psi_{2}$ and $\|\Phi\|_{c b}=\left\|\Psi_{1}(1)+\Psi_{2}(1)\right\|$.

Slice maps. If $A$ and $B$ are $C^{*}$-algebras and $f \in B^{*}$, then

$$
R S_{f}(a \otimes b)=a f(b)
$$

defines a bounded operator $R S_{f}: A \otimes B \rightarrow A$ with norm $\|f\|$, which extends uniquely to a strictly continuous linear map of $M(A \otimes B)$ into $M(A)$. If $f$ is the vector functional $b \rightarrow\langle b \xi, \eta\rangle$ on $\mathscr{B}\left(\mathscr{H}_{2}\right)$, there is also a weakly continuous slice map $R S_{f}: \mathscr{B}\left(\mathscr{H}_{1} \otimes \mathscr{H}_{2}\right) \rightarrow \mathscr{B}\left(\mathscr{H}_{1}\right)$ given by the equation

$$
\left\langle R S_{f}(T) h, k\right\rangle=\langle T(h \otimes \xi), k \otimes \eta\rangle \quad \text { for } h, k \in \mathscr{H}_{1} .
$$


The two kinds of slice map agree when they both make sense-indeed, the second is just the weakly continuous extension of the first (see [14, Lemma 1.5]).

Of course, there are also left slice maps from $A \otimes B$ to $B$, defined by $L S_{f}(a \otimes b)=f(a) b$ for $f \in A^{*}$, and the two kinds of slice maps are related by the Fubini theorem

$$
g \circ L S_{f}=f \circ R S_{g} \text { for } f \in A^{*}, g \in B^{*} .
$$

We shall use this fact in its own right, and in $\S 4$ to give a definition of a slice map for a non-normal functional on a von Neumann algebra. When we imagine no confusion is likely, we shall refer to either $L S_{f}$ or $R S_{f}$ as $S_{f}$.

Group algebras and crossed products. Let $G$ be a locally compact group and $\lambda$ the left regular representation of $G$ on $L^{2}(G)$. The closure of $\left\{\lambda(f): f \in L^{1}(G)\right\}$ in $\mathscr{B}\left(L^{2}(G)\right)$ is the reduced group $C^{*}$ algebra $C_{r}^{*}(G)$, and the weak closure is the group von Neumann algebra $v N(G)$. Let $A(G)$ denote the Fourier algebra of Eymard [7]. Then we have natural identifications $v N(G)=C_{r}^{*}(G)^{* *}=A(G)^{*}$; if $z \in L^{1}(G)$, $f \in A(G)$ then the latter pairing is given by $(f, z)=\int f(s) z(s) d s$. Every such functional has the form $z \rightarrow\langle\lambda(z) \xi, \eta\rangle$ for some $\xi, \eta \in L^{2}(G)$, and a comparison of these formulas shows that every $f \in A(G)$ can be decomposed

$$
f(s)=\overline{(\eta * \tilde{\xi})(s)}=\int \xi\left(s^{-1} r\right) \overline{\eta(r)} d r,
$$

where $\tilde{\xi}(s)=\overline{\xi\left(s^{-1}\right)}$. Most of the time we shall be assuming our group $G$ is amenable, in which case $\lambda$ is an isomorphism of the full group $C^{*}$-algebra $C^{*}(G)$ onto $C_{r}^{*}(G)[21,7.3 .9]$.

By an action of $G$ on a $C^{*}$-algebra $A$ we mean a strongly continuous homomorphism $\alpha: G \rightarrow$ Aut $A$. A covariant representation of $(A, G, \alpha)$ is then a pair $(\pi, U)$ consisting of a (*-preserving) representation $\pi$ of $A$ on a Hilbert space $\mathscr{H}$ and a (strongly continuous) unitary representation $U$ of $G$ on $\mathscr{H}$ such that

$$
\pi\left(\alpha_{s}(a)\right)=U_{s} \pi(a) U_{s}^{*} \text { for } a \in A, s \in G .
$$

The crossed product $A \rtimes_{\alpha} G$ is a $C^{*}$-algebra whose representations are in one-to-one correspondence with the covariant representations of $(A, G, \alpha)$; it is usually constructed as the completion of the algebra $C_{c}(G, A)$ of continuous $A$-valued functions of compact support (see $[21, \S 7.6])$. There are canonical homomorphisms $i_{A}: A \rightarrow M\left(A \rtimes_{\alpha} G\right)$, 
$i_{G}: G \rightarrow U M\left(A \rtimes_{\alpha} G\right)$ such that $i_{A}\left(\alpha_{s}(a)\right)=i_{G}(s) i_{A}(a) i_{G}(s)^{*}$ (see [21, Proof of 7.6.4], or [22] for a different treatment). If we have another action $\beta: G \rightarrow$ Aut $B$, then we shall say a bounded linear map $\Phi: A \rightarrow B$ is $\alpha-\beta$ equivariant (or just equivariant) if $\Phi\left(\alpha_{s}(a)\right)=$ $\beta_{s}(\Phi(a))$ for $s \in G, a \in A$. Thus, for example, if $(\pi, U)$ is a covariant representation of $(A, G, \alpha)$, then $\pi$ is $\alpha$-Ad $U$ equivariant in this sense.

Coactions and their crossed products. Here we shall review some of the basic definitions and results about coactions of a group on a $C^{*}$ algebra, and then discuss what it means for a linear operator between $C^{*}$-algebras to be equivariant with respect to coactions. Our basic reference for this material is $[14, \S 2,3]$.

Loosely speaking, a coaction of a locally compact group $G$ on a $C^{*}$-algebra $A$ is an action of the dual $\hat{G}$ of $G$ on $A$. To make this formal, we first have to introduce the comultiplication $\delta_{G}: C_{r}^{*}(G) \rightarrow$ $M\left(C_{r}^{*}(G) \otimes C_{r}^{*}(G)\right)$, which is the integrated form of the homomorphism $s \mapsto \delta_{s} \otimes \delta_{s}$; spatially, $\delta_{G}$ is given by $\delta_{G}(z)=W_{G}(z \otimes 1) W_{G}^{*}$, where $W_{G} \in U\left(L^{2}(G \times G)\right)$ is defined by $W_{G} \xi(s, t)=\xi\left(s, s^{-1} t\right)$. (This latter formula for $\delta_{G}$ implies that $\delta_{G}$ extends to a homomorphism of $v N(G)$ into $v N(G) \bar{\otimes} v N(G)$.) Then a coaction of $G$ on $A$ is a homomorphism $\delta: A \rightarrow M\left(A \otimes C_{r}^{*}(G)\right)$ satisfying

(1) $\delta(a)(1 \otimes z),(1 \otimes z) \delta(a) \in A \otimes C_{r}^{*}(G)$ for all $a \in A, z \in C_{r}^{*}(G)$;

(2) there is an approximate identity $\left\{e_{j}\right\}$ for $A$ such that $\delta\left(e_{j}\right) \rightarrow 1$ strictly in $M\left(A \otimes C_{r}^{*}(G)\right)$; and

(3) $(\delta \otimes i) \circ \delta=\left(i \otimes \delta_{G}\right) \circ \delta$.

We say $\delta$ is non-degenerate if in addition

(4) for each $\phi \in A^{*}$ there exists $\psi \in C_{r}^{*}(G)^{*}$ such that $(\phi \otimes \psi) \circ \delta \neq 0$. Of these conditions, (1) is technically useful because it implies $S_{f}(\delta(a))$ $\in A$ for $f \in C_{r}^{*}(G)^{*}$, (2) is there to ensure that (3) makes sense, (3) is the analogue for coactions of the multiplicativity $\alpha_{s t}=\alpha_{s} \circ \alpha_{t}$ of an action, and (4) is a technical assumption which is automatic if $G$ is amenable (see [11, Theorem 5] for equivalent formulations of (4)). We mention three examples:

(a) if $G$ is abelian and $\alpha: \hat{G} \rightarrow$ Aut $A$ is an action of $\hat{G}$, then

$$
\delta(a)=\left(\gamma \rightarrow \alpha_{\gamma}(a)\right) \in C_{b}(\hat{G}, A) \subset M\left(A \otimes C_{0}(\hat{G})\right)=M\left(A \otimes C^{*}(G)\right)
$$

defines a coaction of $G$ on $A$;

(b) $\delta_{G}$ is a coaction of $G$ on $C_{r}^{*}(G)$;

(c) there is a canonical coaction $\hat{\alpha}$ of $G$ on the reduced crossed product $A \rtimes_{\alpha, r} G$ (which equals $A \rtimes_{\alpha} G$ if $G$ is amenable).

For a discussion of these examples, see [14, 2.3]. 
A corepresentation of $G$ is a representation of $C_{0}(G)$, which we think of as a representation of $\hat{G}$ (if $G$ is abelian, $C_{0}(G) \cong C^{*}(\hat{G})$ ). A theorem of Nakagami and Takesaki [15, Theorem A.1(b)] gives a oneto-one correspondence between representations $\mu: C_{0}(G) \rightarrow \mathscr{B}(\mathscr{H})$ and unitary operators $W \in \mathscr{B}(\mathscr{H}) \bar{\otimes} v N(G)$ satisfying

$$
(W \otimes 1)(W \otimes 1)^{\sigma}=i \otimes \delta_{G}(W),
$$

where $\sigma$ denotes the flip automorphism of $v N(G) \bar{\otimes} v N(G)$, and $\delta_{G}$ has been extended spatially to $v N(G)$. This correspondence is characterized by the relation

$$
\mu(f)=R S_{f}(W) \text { for } f \in A(G)=v N(G)_{*} .
$$

Strangely, it is often more convenient to work with the unitary $W$ rather than the representation $\mu$, and we refer to $W$ as a corepresentation of $G$ on $\mathscr{H}$.

Associated with a coaction $\delta: A \rightarrow M\left(A \otimes C_{r}^{*}(G)\right)$ is the crossed product $A \rtimes_{\delta} G[14,2.4]$, which is the $C^{*}$-subalgebra of $M\left(A \otimes \mathscr{K}\left(L^{2}(G)\right)\right)$ generated by the elements of the form $\delta(a)\left(1 \otimes M_{f}\right)$ for $a \in A, f \in C_{0}(G)$. Representations of $A \rtimes_{\delta} G$ are in one-to-one correspondence with the covariant representations of $(A, G, \delta)$, each of which consists of a pair $(\pi, W)$ of a representation $\pi$ of $A$ on a space $\mathscr{H}$ and a corepresentation $W$ of $G$ on $\mathscr{H}$ such that

$$
\pi \otimes i(\delta(a))=W(\pi(a) \otimes 1) W^{*} \quad \text { for } a \in A .
$$

The representation of $A \rtimes_{\delta} G$ corresponding to $(\pi, W)$ is denoted $\pi \times W$, and is characterized by the property

$$
\pi \times W\left(\delta(a)\left(1 \otimes M_{f}\right)\right)=\pi(a) R S_{f}(W) \quad \text { for } a \in A, f \in A(G) .
$$

These results are the content of $[14, \S 3]$.

Let $\delta, \varepsilon$ be coactions of $G$ on $C^{*}$-algebras $A, B$ and $\Phi: A \rightarrow B$ be a completely bounded map. We shall say $\Phi$ is $\delta-\varepsilon$ equivariant, or just equivariant, if

$$
(\Phi \otimes i) \circ \delta=\varepsilon \circ \Phi
$$

notice that the left-hand side of this makes sense because completely bounded operators lift through tensoring [5, Lemma 1.5], and extend to the multiplier algebra by Lemma 2.1. Thus, for example, equation (1) says that if $(\pi, W)$ is a covariant representation of $(A, G, \delta)$, then $\pi$ is $\delta-\operatorname{Ad} W$ equivariant. (Here $\operatorname{Ad} W$ denotes the coaction $T \rightarrow$ $W(T \otimes 1) W^{*}$ of $G$ on $\mathscr{B}(\mathscr{H})$.) By using slice maps, we can reformulate 
(2) to avoid appealing to the lifting result: it is equivalent to

$$
\Phi\left(R S_{f}(\delta(a))\right)=R S_{f}(\varepsilon(\Phi(a))) \text { for } a \in A, f \in A(G) \text {. }
$$

This enables us to extend the definition of equivariance to arbitrary bounded operators from $A$ to $B$.

3. Lifting through actions. Let $\alpha: G \rightarrow$ Aut $A$ and $\beta: G \rightarrow$ Aut $B$ be actions of an amenable locally compact group $G$ on $C^{*}$-algebras $A$ and $B$. We shall prove that an equivariant completely bounded map $\Phi: A \rightarrow B$ gives rise to a completely bounded map $\Phi \times i: A \rtimes_{\alpha} G \rightarrow$ $B \rtimes_{\beta} G$. We shall deduce this from a structure theorem for equivariant completely bounded maps $\Phi: A \rightarrow \mathscr{B}(\mathscr{H})$ (Theorem 3.3), for which we need equivariant versions of Stinespring's theorem (Lemma 3.1) and Wittstock's decomposition theorem (Lemma 3.2). Lemma 3.1 is essentially proved in [17], so we shall merely outline its proof.

LemMa 3.1. Let $\Phi:(A, G, \alpha) \rightarrow(\mathscr{B}(\mathscr{H}), G, \operatorname{Ad} U)$ be an equivariant completely positive map. Then there are

(1) a covariant representation $(\pi, W)$ of $(A, G, \alpha)$ on a space $\mathscr{K}$,

(2) a continuous operator $V: \mathscr{H} \rightarrow \mathscr{K}$, and

(3) a representation $\rho$ of the commutant $\Phi(A)^{\prime}$ on $\mathscr{K}$, such that

(a) $\Phi(a)=V^{*} \pi(a) V$ for $a \in A$,

(b) $\mathscr{K}=(\pi(A) V \mathscr{H})^{-}$,

(c) $V U_{t}=W_{t} V$ for all $t \in G$, and

(d) $\rho(x) V=V x$ for $x \in \Phi(A)^{\prime}, \rho\left(U_{t} x U_{t}^{*}\right)=W_{t} \rho(x) W_{t}^{*}$ for $x \in$ $\Phi(A)^{\prime}, t \in G$, and $\rho\left(\Phi(A)^{\prime}\right) \subseteq \pi(A)^{\prime}$.

Outline of Proof. (See [24, pp. 195-196] and [17, Theorem 2.1].) We define a pre-inner product on the algebraic tensor product $A \odot \mathscr{H}$ by

$$
\langle a \otimes \xi, b \otimes \eta\rangle=\left\langle\Phi\left(b^{*} a\right) \xi, \eta\right\rangle,
$$

mod out by the vectors of length 0 , and take $\mathscr{H}$ to be the completion. Then we set $\pi(a)(b \otimes \xi)=a b \otimes \xi, \rho(x)(b \otimes \xi)=b \otimes x \xi$, $W_{t}(a \otimes \xi)=\alpha_{t}(a) \otimes U_{t} \xi$ and, if $A$ has an identity, $V \xi=1 \otimes \xi$; otherwise $V \xi$ is defined as the norm limit of $e_{\gamma} \otimes \xi$, where $e_{\gamma}$ runs through an approximate identity for $A$. The standard arguments show that (a), (b) and the first part of (d) hold [24, p. 195]. The equivariance of $\Phi$ implies that $W_{t}$ is isometric, and it has inverse $W_{t^{-1}}$, so each $W_{t}$ is a unitary operator on $\mathscr{K} ; W$ is a homomorphism because $\alpha$ and 
$U$ are, and it follows from a standard inequality that $W$ is strongly continuous. Condition (c) is trivial when $A$ has an identity, and in general is a consequence of the fact that the operator $V$ is the same no matter which approximate identity we use. The other two parts of (d) are easily checked on elementary tensors, and this completes the proof.

LemMa 3.2. If $\Phi:(A, G, \alpha) \rightarrow(\mathscr{B}(\mathscr{H}), G, \operatorname{Ad} U)$ is a symmetric equivariant completely bounded operator, then there are completely positive operators $\Phi_{j}: A \rightarrow \mathscr{B}(\mathscr{H})$ such that $\Phi=\Phi_{1}-\Phi_{2}$ and $\|\Phi\|_{c b} \geq$ $\left\|\Phi_{1}(1)+\Phi_{2}(1)\right\|$.

Proof. By the usual Wittstock theorem ([25], [19]) there are completely positive operators $\Psi_{j}: A \rightarrow \mathscr{B}(\mathscr{H})$ such that $\Phi=\Psi_{1}-\Psi_{2}$ and $\|\Phi\|_{c b}=\left\|\Psi_{1}(1)+\Psi_{2}(1)\right\|$. (In the non-unital case, the identity here is in $M(A)$.) Let $m$ be a right invariant mean on $G$, and define $\Phi_{j}$ by

$$
\left\langle\Phi_{j}(a) \xi, \eta\right\rangle=m\left(t \mapsto\left\langle U_{t}^{*} \Psi_{j}\left(\alpha_{t}(a)\right) U_{t} \xi, \eta\right\rangle\right) \text { for } a \in A, \xi, \eta \in \mathscr{H} ;
$$

here the right-hand side is a bounded sesquilinear form in $\xi$ and $\eta$, and hence defines a bounded operator $\Phi_{j}(a)$ on $\mathscr{H}$. The complete positivity of $\Phi_{j}$ follows from that of $\Psi_{j}$ and the positivity of $m$. The right invariance $m(t \mapsto f(t s))=m(f)$ shows that $\Phi_{j}\left(\alpha_{s}(a)\right)=U_{s} \Phi_{j}(a) U_{s}^{*}$ for $a \in A, s \in G$, and the equivariance of $\Phi$ implies that $\Phi=\Phi_{1}-\Phi_{2}$. The inequality $\left\|\Phi_{1}(1)+\Phi_{2}(1)\right\| \leq\|\Phi\|_{c b}$ can be obtained by averaging the equation

$$
\left\|U_{s}^{*} \Psi_{1}(1) U_{s}+U_{s}^{*} \Psi_{2}(1) U_{s}\right\|=\|\Phi\|_{c b},
$$

and this completes the proof of the lemma.

THEOREM 3.3. Let $\alpha$ be an action of a locally compact amenable group $G$ on a $C^{*}$-algebra $A$, and let $U$ be a unitary representation of $G$ on a Hilbert space. If $\Phi: A \rightarrow \mathscr{B}(\mathscr{H})$ is a completely bounded equivariant linear map, then there are

(1) a covariant representation $(\pi, W)$ of $(A, G, \alpha)$ on a Hilbert space $\mathscr{K}$, and

(2) bounded operators $V: \mathscr{H} \rightarrow \mathscr{K}, T: \mathscr{K} \rightarrow \mathscr{H}$ such that

(a) $\Phi(a)=T \pi(a) V$ for $a \in A$, 
(b) $V U_{t}=W_{t} V, T W_{t}=U_{t} T$ for $t \in G$, and

(c) $\|\Phi\|_{c b}=\|T\|\|V\|$.

Proof. We begin by observing that it will be enough to prove the result for a symmetric operator $\Phi$. To see this, we introduce $\Phi: A \rightarrow$ $\mathscr{B}(\mathscr{H} \oplus \mathscr{H})$ defined by

$$
\Phi(a)=\left(\begin{array}{cc}
0 & \Phi(a) \\
\Phi\left(a^{*}\right)^{*} & 0
\end{array}\right) \quad \text { for } a \in A .
$$

Then $\Phi$ is a symmetric completely bounded operator, and a calculation using the equality

$$
\left\|\left(\begin{array}{ll}
0 & x \\
y & 0
\end{array}\right)\right\|=\max \{\|x\|,\|y\|\}
$$

shows that $\|\underline{\Phi}\|_{c b}=\|\Phi\|_{c b}$. Define $\underline{U}=U \oplus U$, so that $\underline{\Phi}$ is $\alpha-\operatorname{Ad} \underline{U}$ equivariant, let $T_{0}: \mathscr{H} \oplus \mathscr{H} \rightarrow \mathscr{H}$ be the projection onto the first factor, and let $V_{0}: \mathscr{H} \rightarrow \mathscr{H} \oplus \mathscr{H}$ be the inclusion of the second factor. Then if $\Phi(a)=T \pi(a) V$ is a realization of $\Phi$ satisfying (a)-(c), the required realization of $\Phi$ is given by $\Phi(a)=T_{0} T \pi(a) V V_{0}$.

Now suppose that $\Phi$ is symmetric, and let $\Phi=\Phi_{1}-\Phi_{2}$ be the decomposition of Lemma 3.2. By Lemma 3.1 there are covariant representations $\left(\pi_{j}, W_{j}\right)$ of $(A, G, \alpha)$ on $\mathscr{K}_{j}$ and operators $V_{j}: \mathscr{H} \rightarrow \mathscr{K}_{j}$ satisfying $\Phi_{j}(a)=V_{j}^{*} \pi(a) V_{j}$ (and the other conditions of 3.1). We then take $\mathscr{K}=\mathscr{K}_{1} \oplus \mathscr{K}_{2}, \pi=\pi_{1} \oplus \pi_{2}, W=W_{1} \oplus W_{2}, V=\left(V_{1},-V_{2}\right)^{\text {tr }}$, and $T=\left(V_{1}^{*}, V_{2}^{*}\right)$. Properties (a) and (b) follow immediately, and we have

$$
\|T\|\|V\|=\left\|V_{1}^{*} V_{1}+V_{2}^{*} V_{2}\right\|=\left\|\Phi_{1}(1)+\Phi_{2}(1)\right\| \leq\|\Phi\|_{c b} .
$$

But any decomposition $\Phi(a)=T \pi(a) V$ implies that $\|\Phi\|_{c b} \leq\|T\|\|V\|$ [4, Lemma 5.3], so this completes the proof of the theorem.

REMARK 3.4. If there is an equivariant version of Arveson's extension theorem [1] which works for an arbitrary locally compact group, then the techniques of [19] would enable us to remove the hypothesis of amenability from Theorem 3.3. For another possible route to the same end, see the proof of [8, Theorem 3.7].

THEOREM 3.5. Let $G$ be an amenable locally compact group, and let $\alpha$ and $\beta$ be actions of $G$ on $C^{*}$-algebras $A$ and $B$ respectively. If $\Phi: A \rightarrow B$ is a completely bounded equivariant operator, then there is a unique completely bounded operator $\Phi \times i: A \rtimes_{\alpha} G \rightarrow B \rtimes_{\beta} G$ such 
that

(a) $\Phi \times i\left(i_{A}(a) i_{G}(z)\right)=i_{B}(\Phi(a)) i_{G}(z)$ for $a \in A, z \in C_{c}(G)$.

We then also have

(b) $(\Phi \times i(y))(t)=\Phi(y(t))$ for $y \in C_{c}(G, A), t \in G$;

(c) $\|\Phi \times i\|_{c b}=\|\Phi\|_{c b}$;

(d) $\Phi \times i$ completely positive if $\boldsymbol{\Phi}$ is, and symmetric if $\boldsymbol{\Phi}$ is;

(e) $\Phi \times i$ is equivariant for the dual coactions $\hat{\alpha}$ and $\hat{\beta}$ of $G$.

Proof. Let $(\rho, U)$ be a covariant representation of $(B, G, \beta)$ on $\mathscr{H}$ such that $\rho \times U$ is a faithful representation of $B \rtimes_{\beta} G$. Then $\rho \circ$ $\Phi$ is an equivariant completely bounded operator from $(A, G, \alpha)$ to $(\mathscr{B}(\mathscr{H}), G, \operatorname{Ad} U)$, so we can apply Theorem 3.3 to realize $\rho \circ \Phi$ in the form $\rho \circ \Phi(a)=T \pi(a) V$, where $\pi, T, V, \mathscr{K}, W$ are as in that theorem. Then for each $a \in A, z \in C_{c}(G)$ we have

$$
\begin{aligned}
T\left(\pi \times W\left(i_{A}(a) i_{G}(z)\right)\right) V & =T \pi(a) W(z) V=T \pi(a) V U(z) \\
& =\rho \circ \Phi(a) U(z)=\rho \times U\left(i_{B}(\Phi(a)) i_{G}(z)\right) .
\end{aligned}
$$

Thus $y \mapsto T(\pi \times W(y)) V$ maps $A \rtimes_{\alpha} G$ into the range of $\rho \times U$, and we can define

$$
\Phi \times i(y)=(\rho \times U)^{-1}(T(\pi \times W(y)) V) \text { for } y \in A \rtimes_{\alpha} G .
$$

Then $\Phi \times i$ is a completely bounded map with

$$
\|\Phi \times i\|_{c b} \leq\|T\|\|V\|=\|\Phi\|_{c b},
$$

and the above calculation shows that (a) holds. Since the elements of the form $i_{A}(a) i_{G}(z)$ span a dense subspace of $A \rtimes_{\alpha} G$, there is only one continuous operator satisfying (a). Note that the strictly continuous extension $\Psi$ of $(\rho \times U) \circ(\Phi \times i)$ satisfies

$$
\Psi\left(i_{A}(a)\right)=T\left(\pi \times W\left(i_{A}(a)\right)\right) V=T \pi(a) V=\rho(\Phi(a)) \in \rho(B),
$$

so $\Phi=\rho^{-1} \circ \Psi \circ i_{A}$. But $\Psi$ has the same completely bounded norm as $\Phi \times i$, so this implies $\|\Phi\|_{c b} \leq\|\Psi\|_{c b}=\|\Phi \times i\|_{c b}$, which gives (c).

To establish (b), we approximate $z$ uniformly on $\operatorname{supp} z$ by a sum $\sum i_{A}\left(a_{j}\right) i_{G}\left(z_{j}\right)$ where $\operatorname{supp} z_{j} \subset \operatorname{supp} z$. Since $\Phi$ is continuous, $\sum i_{B}\left(\Phi\left(a_{j}\right)\right) i_{G}\left(z_{j}\right)$ uniformly approximates $\Phi \circ z$, and the continuity of $\Phi \times i$ gives

$$
\begin{aligned}
\Phi \times i(z) & \sim \sum_{j} \Phi \times i\left(i_{A}\left(a_{j}\right) i_{G}\left(z_{j}\right)\right) \\
& =\sum_{j} i_{B}\left(\Phi\left(a_{j}\right)\right) i_{G}\left(z_{j}\right) \sim \Phi \circ z .
\end{aligned}
$$


This implies (b). If $\Phi$ is completely positive, we can choose $T=V^{*}$ (see Lemma 3.1), and then $\Phi \times i$ is also completely positive. Since a completely bounded operator is symmetric if and only if it is the difference of completely positive operators, this also shows that $\Phi \times i$ is symmetric if $\Phi$ is. This gives (d), and it only remains to verify (e).

We shall show that

$$
\Phi \times i\left(S_{f}(\hat{\alpha}(z))\right)=S_{f}(\hat{\beta}(\Phi \times i(z))) \text { for all } f \in A(G), z \in C_{c}(G, A),
$$

and we begin by showing that $S_{f}(\hat{\alpha}(z))$ is the pointwise product of the function $f$ and $z$. Choose a faithful representation $\rho$ of $A$ on $\mathscr{H}$; then $A \rtimes_{\alpha} G$ is faithfully represented on $L^{2}(G, \mathscr{H})$ via $\tilde{\rho} \times \lambda[21,7.7 .5]$ and $\hat{\alpha}(z)$ is given on $L^{2}(G \times G, \mathscr{H})$ by

$$
(\hat{\alpha}(z) \nu)(t, r)=\int \rho\left(\alpha_{t}^{-1}(z(s))\right)\left(\nu\left(s^{-1} t, s^{-1} r\right)\right) d s
$$

(see [13, p. 155]). Let $\xi, \eta \in L^{2}(G)$ be two vectors such that $(f, y)=$ $\langle y \xi, \eta\rangle$ for all $y \in C_{r}^{*}(G)$. Then for $h, k \in L^{2}(G, \mathscr{H})$ and $z \in C_{c}(G, A)$ we have

$$
\begin{aligned}
\langle(\tilde{\rho} \times & \left.\lambda)\left(S_{f}(\hat{\alpha}(z))\right) h, k\right\rangle=\langle\hat{\alpha}(z)(h \otimes \xi),(k \otimes \eta)\rangle \\
= & \iint\left\{\int \rho\left(\alpha_{t}^{-1}(z(s))\right)\left(h\left(s^{-1} t\right) \xi\left(s^{-1} r\right)\right) d s\right\} \overline{k(t) \eta(r)} d t d r \\
= & \iint \rho\left(\alpha_{t}^{-1}\left(z(s) \int \xi\left(s^{-1} r\right) \overline{\eta(r)} d r\right)\right) h\left(s^{-1} t\right) \overline{k(t)} d s d t \\
= & \int \tilde{\rho} \times \lambda(f \cdot z)(h)(t) \overline{k(t)} d t \\
= & \langle\tilde{\rho} \times \lambda(f \cdot z) h, k\rangle .
\end{aligned}
$$

Thus $S_{f}(\hat{\alpha}(z))=f \cdot z$, as claimed. Now we have by (b) that

$$
\begin{gathered}
(\Phi \times i)\left(S_{f}(\hat{\alpha}(z))\right)(s)=\Phi(f(s) z(s))=f(s) \Phi(z(s)) \\
=f(s) \Phi \times i(z)(s)=S_{f}(\hat{\beta}(\Phi \times i(z)))(s)
\end{gathered}
$$

for all $s \in G, z \in C_{c}(G, A)$ and $f \in A(G)$. This completes the proof of (e).

4. Lifting through coactions. The basic structure of this section is similar to that of $\S 3$ with the actions of that section replaced by coactions, although the results and calculations become technically more complicated. One underlying difficulty is that, given a coaction $\delta$ of $G$ on $A$, there is at present no nice *-algebra whose completion is $A \rtimes_{\delta} G$ in the way that $A \rtimes_{\alpha} G$ is the completion of $C_{c}(G, A)$ with a suitable product and norm. At several points in the argument we 
shall split off technical sublemmas to deal with some tricky calculation. Certain calculations will involve approximations arising from the use of approximate identities: to simplify the notation and avoid explicit inequalities we shall denote the approximation by $\sim$ in these cases.

Theorem 4.1 is a covariant Stinespring result for coactions corresponding to Lemma 3.1 for actions, and Theorem 4.5 an equivariant version of the Wittstock decomposition analogous to Lemma 3.2.

THEOREM 4.1. Let $G$ be a locally compact group, let $\delta: A \rightarrow$ $M\left(A \otimes C_{r}^{*}(G)\right)$ be a nondegenerate coaction of $G$ on a $C^{*}$-algebra $A$, and let $W \in \mathscr{B}\left(\mathscr{H} \otimes L^{2}(G)\right)$ be a corepresentation of $G$ on $\mathscr{H}$. If $\Phi: A \rightarrow \mathscr{B}(\mathscr{H})$ is a $\delta$ - Ad $W$ equivariant completely positive linear operator, then there are

(1) a covariant representation $(\pi, Y)$ of $(A, G, \delta)$ on a Hilbert space $\mathscr{K}$,

(2) a continuous linear operator $V: \mathscr{H} \rightarrow \mathscr{K}$, and

(3) a representation $\rho$ of $\Phi(A)^{\prime}$ on $\mathscr{K}$, such that

(a) $\Phi(a)=V^{*} \pi(a) V$,

(b) $\mathscr{K}=(\pi(A) V \mathscr{H})^{-}$,

(c) $(V \otimes 1) W=Y(V \otimes 1)$ and $\left(V^{*} \otimes 1\right) Y=W\left(V^{*} \otimes 1\right)$,

(d) $\rho(x) V=V x, W^{*}(x \otimes 1) W \in(\Phi(A) \otimes 1)^{\prime}$, and

$$
(\rho \otimes i)\left(W^{*}(x \otimes 1) W\right)=Y^{*}(\rho(x) \otimes 1) Y
$$

for all $a \in A$ and $x \in \Phi(A)^{\prime}$.

Proof. Let $N$ be the subspace of the pre-inner product space $A \odot \mathscr{H}$ of vectors of zero length, where the pre-inner product is defined by $\langle a \otimes h, b \otimes k\rangle=\left\langle\Phi\left(b^{*} a\right) h, k\right\rangle$ as usual. Then $\mathscr{K}$ is the completion of $A \odot \mathscr{H} / N$, and $\pi, \rho$, and $V$ are defined in the usual way (see Lemma 3.1). We shall define a unitary operator $Y$ on $\mathscr{K} \otimes L^{2}(G)$ by

$$
Y(a \otimes h \otimes \xi)=(\pi \otimes i)(\delta(a))(V \otimes 1(W(h \otimes \xi)))
$$

for all $a \in A, h \in H$ and $\xi \in L^{2}(G)$, where we are viewing $C_{r}^{*}(G)$ as acting concretely on $L^{2}(G)$. We shall show that this equation does define a unitary operator $Y$ by verifying

(i) $Y$ is a well-defined operator on the algebraic tensor product $A \odot\left(\mathscr{H} \odot L^{2}(G)\right)$

(ii) $N \odot L^{2}(G) \subseteq \operatorname{ker} Y$,

(iii) $Y$ is isometric from $((A \odot \mathscr{H}) / N) \odot L^{2}(G)$ into $\mathscr{H} \otimes L^{2}(G)$, and

(iv) $Y$ is surjective when extended to $\mathscr{K} \otimes L^{2}(G)$. 
We shall then prove that $Y$ is a corepresentation and that (c) and (d) hold. The conclusions not involving $Y$ are standard Stinespring theory.

We begin by noting that $Y$ is the composition

$$
\begin{array}{rr}
A \odot \mathscr{H} \odot L^{2}(G) \stackrel{\delta \otimes W}{\longrightarrow} M\left(A \otimes C_{r}^{*}(G)\right) \odot\left(\mathscr{H} \otimes L^{2}(G)\right) \\
& \downarrow(\pi \otimes i) \otimes V \otimes i \\
\mathscr{B}\left(\mathscr{K} \otimes L^{2}(G)\right) \odot\left(\mathscr{K} \otimes L^{2}(G)\right) \stackrel{\text { eval }}{\longrightarrow} & \mathscr{K} \otimes L^{2}(G),
\end{array}
$$

where for a Hilbert space $\mathscr{L}$, eval: $\mathscr{B}(\mathscr{L}) \odot \mathscr{L} \rightarrow \mathscr{L}$ is the linear map such that eval $(T \otimes l)=T(l)$. Thus $Y$ is a composition of linear operators and hence is a well-defined linear operator; this proves (i). To obtain (ii) and (iii) we need a couple of lemmas.

LEMMA 4.2. If $f=(\eta * \tilde{\xi})^{-} \in A(G)$ with $\eta, \xi \in L^{2}(G)$, then

$$
\langle(\pi \otimes i)(m)(b \otimes h \otimes \xi), c \otimes k \otimes \eta\rangle=\left\langle\Phi\left(c^{*} S_{f}(m) b\right) h, k\right\rangle
$$

for all $m \in M\left(A \otimes C_{r}^{*}(G)\right), b, c \in A$, and $h, k \in \mathscr{K}$.

Proof. First we suppose that $m=a \otimes z \in A \odot L^{1}(G)$. Then the left-hand side is

$$
\begin{aligned}
\langle a b \otimes h \otimes z \xi, c \otimes k \otimes \eta\rangle & =\langle a b \otimes h, c \otimes k\rangle\langle z \xi, \eta\rangle \\
& =\left\langle\Phi\left(c^{*} a b\right) h, k\right\rangle(z, f)
\end{aligned}
$$

because the pairing between $A(G)$ and $C_{r}^{*}(G)$ is given by

$$
\begin{aligned}
& (z, f)=\langle z \xi, \eta\rangle \text { if } f=(\eta * \tilde{\xi})^{-}, \\
= & \left\langle\Phi\left(c^{*} a(z, f) b\right) h, k\right\rangle \\
= & \left\langle\Phi\left(c^{*} S_{f}(a \otimes z) b\right) h, k\right\rangle .
\end{aligned}
$$

This formula extends to $A \otimes C_{r}^{*}(G)$ by continuity, and then to $M\left(A \otimes C_{r}^{*}(G)\right)$ by strict continuity.

LemMA 4.3. If $a, b \in A, h, k \in \mathscr{H}$, and $\xi, \eta \in L^{2}(G)$, then $\langle Y(a \otimes h \otimes \xi), Y(b \otimes k \otimes \eta)\rangle=\langle a \otimes h, b \otimes k\rangle\langle\xi, \eta\rangle$.

Proof. By definition we have

$I=\langle Y(a \otimes h \otimes \xi), Y(b \otimes k \otimes \eta)\rangle$

$=\langle\pi \otimes i(\delta(a))(V \otimes 1(W(h \otimes \xi))), \pi \otimes i(\delta(b))(V \otimes 1(W(k \otimes \eta)))\rangle$. 
We can approximate $W(h \otimes \xi)$ and $W(k \otimes \eta)$ by finite sums $\sum h_{j} \otimes \xi_{j}$ and $\sum k_{j} \otimes \eta_{j}$ in $\mathscr{H} \odot L^{2}(G)$. Let $f_{j r}$ denote the functional in $v N(G)_{*}=$ $A(G)$ defined by

$$
\left(g, f_{j r}\right)=\left\langle g \xi_{j}, \eta_{r}\right\rangle
$$

for $g \in L^{1}(G) \subseteq C_{r}^{*}(G)$. Let $e_{\gamma}$ be a bounded approximate identity for $A$ and let $V_{\gamma}: \mathscr{H} \rightarrow \mathscr{K}$ be defined by $V_{\gamma}(h)=e_{\gamma} \otimes h$. Then the operator $V$ occurring in the Stinespring decomposition is the strong operator limit of the $V_{\gamma}$; this is how $V$ is defined when $A$ does not have an identity [24, p. 197]. Choose $\gamma$ large enough to ensure that $V_{\gamma}\left(h_{j}\right)$ is near $V\left(h_{j}\right), V_{\gamma}\left(k_{r}\right)$ is near $V\left(k_{r}\right)$, and $e_{\gamma}^{*} S_{f_{j r}}\left(\delta\left(b^{*} a\right)\right) e_{\gamma}$ is near $S_{f_{j r}}\left(\delta\left(b^{*} a\right)\right)$ for all $j$ and $r$. Then

$$
\begin{aligned}
I \sim\left\langle\pi \otimes i(\delta(a))\left(V_{\gamma} \otimes 1\left(\sum_{j} h_{j} \otimes \xi_{j}\right)\right),\right. & \left.\pi \otimes i(\delta(b))\left(V_{\gamma} \otimes 1\left(\sum_{r} k_{r} \otimes \eta_{r}\right)\right)\right\rangle \\
= & \sum_{j, r}\left\langle\pi \otimes i(\delta(a))\left(e_{\gamma} \otimes h_{j} \otimes \xi_{j}\right), \pi \otimes i(\delta(b))\left(e_{\gamma} \otimes k_{r} \otimes \eta_{r}\right)\right\rangle,
\end{aligned}
$$

which by Lemma 4.2 equals

$$
\sum_{j, r}\left\langle\Phi\left(e_{\gamma}^{*}\left(S_{f_{j r}}\left(\delta\left(b^{*} a\right)\right) e_{\gamma}\right) h_{j}, j_{r}\right\rangle\right.
$$

$I$ is therefore close to

$$
\sum_{j, r}\left\langle\Phi\left(S_{f_{r r}}\left(\delta\left(b^{*} a\right)\right)\right) h_{j}, k_{r}\right\rangle
$$

Since $\Phi$ is equivariant,

$$
\begin{aligned}
I & \sim \sum_{j, r}\left\langle S_{f_{j r}}\left(W\left(\Phi\left(b^{*} a\right) \otimes 1\right) W^{*}\right) h_{j}, k_{r}\right\rangle \\
& =\sum_{j, r}\left\langle W\left(\Phi\left(b^{*} a\right) \otimes 1\right) W^{*}\left(h_{j} \otimes \xi_{j}\right), k_{r} \otimes \eta_{r}\right\rangle \\
& =\left\langle W\left(\Phi\left(b^{*} a\right) \otimes 1\right) W^{*}\left(\sum_{j} h_{j} \otimes \xi_{j}\right), \sum_{r} k_{r} \otimes \eta_{r}\right\rangle \\
& \sim\left\langle W\left(\Phi\left(b^{*} a\right) \otimes 1\right) W^{*} W(h \otimes \xi), W(k \otimes \eta)\right\rangle \\
& =\left\langle\left(\Phi\left(b^{*} a\right) \otimes 1\right)(h \otimes \xi), k \otimes \eta\right\rangle \\
& =\langle a \otimes h, b \otimes k\rangle\langle\xi, \eta\rangle .
\end{aligned}
$$


The various approximations involved can be made arbitrarily accurate, so Lemma 4.3 follows.

Proof of Theorem 4.1 (continued). It follows from Lemma 4.3 that if $\sum d_{j} \otimes \xi_{j} \in(A \odot \mathscr{H}) \odot L^{2}(G)$, then

$$
\begin{array}{r}
\left\langle\left(\sum_{j} d_{j} \otimes \xi_{j}\right), Y\left(\sum_{j} d_{j} \otimes \xi_{j}\right)\right\rangle=\sum_{j, r}\left\langle Y\left(d_{j} \otimes \xi_{j}\right), Y\left(d_{r} \otimes \xi_{r}\right)\right\rangle \\
=\sum_{j, r}\left\langle d_{j}, d_{r}\right\rangle\left\langle\xi_{j}, \xi_{r}\right\rangle=\left\|\sum_{j} d_{j} \otimes \xi_{j}\right\|^{2} .
\end{array}
$$

Thus $Y$ is a well-defined isometry on $(A \odot \mathscr{H}) / \mathscr{N} \odot L^{2}(G)$; this establishes (ii) and (iii).

Since $Y$ is an isometry, it will be surjective if it has dense range. Thus to prove (iv) it will be enough to approximate an arbitrary elementary tensor by something in the range of $Y$. So fix $a \otimes h \otimes \xi \epsilon$ $A \odot \mathscr{H} \odot L^{2}(G)$. Choose $\gamma$ such that $a e_{\gamma} \sim a$ and $V_{\gamma}(h)=e_{\gamma} \otimes h \sim V h$, and factor $\xi=z \cdot \eta$ for $z \in C_{r}^{*}(G)$ and $\eta \in L^{2}(G)$. Then

$$
\begin{aligned}
a \otimes h \otimes \xi & \sim a e_{\gamma} \otimes h \otimes z \cdot \eta=(\pi(a) \otimes z)\left(e_{\gamma} \otimes h \otimes \eta\right) \\
& =(\pi \otimes i)(a \otimes z)\left(V_{\gamma} \otimes 1(h \otimes \eta)\right) .
\end{aligned}
$$

Since the coaction $\delta$ is non-degenerate, the span of $\delta(A) \cdot\left(A \otimes C_{r}^{*}(G)\right)$ is dense in $A \otimes C_{r}^{*}(G)$ [11, Theorem 5], so $a \otimes z$ can be approximated by a finite sum $\sum \delta\left(a_{j}\right)\left(1 \otimes z_{j}\right)$. Thus

$$
\begin{aligned}
a \otimes h \otimes \xi & \sim \sum_{j} \pi \otimes i\left(\delta\left(a_{j}\right)\left(1 \otimes z_{j}\right)\right)\left(V_{\gamma}(h) \otimes \eta\right) \\
& \sim \sum_{j} \pi \otimes i\left(\delta\left(a_{j}\right)\right)\left(V(h) \otimes z_{j} \cdot \eta\right) \\
& \sim \sum_{j} Y\left(a_{j} \otimes W^{*}\left(h \otimes z_{j} \cdot \eta\right)\right)
\end{aligned}
$$

so the range of $Y$ is dense and (iv) follows. We have proved that $Y$ is unitary.

To show that $Y$ is a corepresentation of $G$ on $\mathscr{K}$ we shall check that $Y$ satisfies the identity $(Y \otimes 1)(1 \otimes \sigma)(Y \otimes 1)(1 \otimes \sigma)=i \otimes \delta_{G}(Y)$ on $\mathscr{K} \otimes L^{2}(G) \otimes L^{2}(G)$, where $\sigma$ denotes the flip $\sigma(\xi \otimes \eta)=\eta \otimes \xi$. Applying the left-hand side to an elementary tensor $a \otimes h \otimes \xi \otimes \eta$ in $(A \odot \mathscr{H}) \odot L^{2}(G) \otimes L^{2}(G)$ gives

$$
\begin{aligned}
L & =(Y \otimes 1)(1 \otimes \sigma)(Y \otimes 1)(1 \otimes \sigma)(a \otimes h \otimes \xi \otimes \eta) \\
& =(Y \otimes 1)(1 \otimes \sigma)\{\pi \otimes i(\delta(a))(V \otimes 1(W(h \otimes \eta))) \otimes \xi\} .
\end{aligned}
$$


The element $W(h \otimes \eta)$ in $H \otimes L^{2}(G)$ can be approximated by a finite tensor $\sum h_{j} \otimes \eta_{j}^{\prime}$; then each $\eta_{j}^{\prime}$ can be factorized $\eta_{j}^{\prime}=z_{j} \cdot \eta_{j}$ for some $z_{j} \in C_{r}^{*}(G)$ and $\eta_{j} \in L^{2}(G)$. Then, for each $j, \delta(a)\left(1 \otimes z_{j}\right) \in A \otimes$ $C_{r}^{*}(G)$, and hence can be approximated by a finite tensor $\sum_{r} a_{j r} \otimes y_{j r}$. We now choose $\gamma$ large enough to ensure that $V_{\gamma} h_{j}=e_{\gamma} \otimes h_{j}$ is near $V h_{j}$ for all $j$ and $a_{j r} e_{j}$ is near $a_{j r}$ for all $j, r$. Then

$$
\begin{gathered}
L \sim(Y \otimes 1)(1 \otimes \sigma)\left\{(\pi \otimes i)(\delta(a))\left(V \otimes 1 \sum_{j} h_{j} \otimes z_{j} \eta_{j}\right) \otimes \xi\right\} \\
\sim \sum_{j}(Y \otimes 1)(1 \otimes \sigma)\left\{\pi \otimes i\left(\delta(a)\left(1 \otimes z_{j}\right)\right)\left(e_{\gamma} \otimes h_{j} \otimes \eta_{j}\right) \otimes \xi\right\} \\
\sim \sum_{j, r}(Y \otimes 1)(1 \otimes \sigma)\left\{\pi \otimes i\left(a_{j r} \otimes y_{j r}\right)\left(e_{\gamma} \otimes h_{j} \otimes \eta_{j}\right) \otimes \xi\right\} \\
=\sum_{j, r}(Y \otimes 1)(1 \otimes \sigma)\left\{a_{j r} e_{\gamma} \otimes h_{j} \otimes y_{j r} \eta_{j} \otimes \xi\right\} \\
=\sum_{j, r} \pi \otimes i\left(\delta\left(a_{j r} e_{\gamma}\right)\right)\left\{V \otimes 1\left(W\left(h_{j} \otimes \xi\right)\right)\right\} \otimes y_{j r} \eta_{j} \\
\sim \sum_{j, r}\left(\pi \otimes i\left(\delta\left(a_{j r}\right)\right) \otimes 1\right)\left(1 \otimes 1 \otimes y_{j r}\right) \\
\quad \times\left(V \otimes 1 \otimes 1\left(W \otimes 1\left(h_{j} \otimes \xi \otimes \eta_{j}\right)\right)\right) \\
=\sum_{j}\left(\pi \otimes i \otimes i\left(\delta \otimes i\left(\sum_{r} a_{j r} \otimes y_{j r}\right)\right)\right) \\
\quad \times\left(V \otimes 1 \otimes 1\left(W \otimes 1\left(h_{j} \otimes \xi \otimes \eta_{j}\right)\right)\right) \\
\sim \sum_{j}\left(\pi \otimes i \otimes i\left(\delta \otimes i(\delta(a))\left(1 \otimes 1 \otimes z_{j}\right)\right)\right) \\
\quad \times\left(V \otimes 1 \otimes 1\left(W \otimes 1\left(h_{j} \otimes \xi \otimes \eta_{j}\right)\right)\right) \\
=\sum_{j}\left(\pi \otimes i \otimes i\left(\left(i \otimes \delta_{G}\right) \circ \delta(a)\right)\right) \\
\times\left(V \otimes 1 \otimes 1\left(W \otimes 1\left(1 \otimes \sigma\left(h_{j} \otimes z_{j} \eta_{j} \otimes \xi\right)\right)\right)\right)
\end{gathered}
$$

on applying the coaction identity $(\delta \otimes i) \circ \delta=\left(i \otimes \delta_{G}\right) \circ \delta$. Continuing this calculation gives

$L \sim \pi \otimes i \otimes i\left(\left(i \otimes \delta_{G}\right) \circ \delta(a)\right)((V \otimes 1 \otimes 1)(W \otimes 1)(1 \otimes \sigma)$

$=\pi \otimes i \otimes i\left(\left(i \otimes \delta_{G}\right) \circ \delta(a)\right)\left((V \otimes 1 \otimes 1) i \otimes \delta_{G}(W)(1 \otimes \sigma)(h \otimes \eta \otimes \xi)\right)$

$=i \otimes \delta_{G}(\pi \otimes i(\delta(a)))\left(V \otimes 1 \otimes 1\left(i \otimes \delta_{G}(W)\right)(h \otimes \xi \otimes \eta)\right)$. 
Applying the right-hand side of the corepresentation identity for $Y$ to the same elementary tensor yields

$$
\begin{aligned}
i \otimes \delta_{G} & (Y)(a \otimes h \otimes \xi \otimes \eta) \\
& =\left(1 \otimes W_{G}\right)(Y \otimes 1)\left(1 \otimes W_{G}^{*}\right)((a \otimes h) \otimes \xi \otimes \eta) \\
= & \left(1 \otimes W_{G}\right)(Y \otimes 1)\left(a \otimes h \otimes W_{G}^{*}(\xi \otimes \eta)\right) \\
= & \left(1 \otimes W_{G}\right)(\pi \otimes i(\delta(a)) \otimes 1)\{(V \otimes 1 \otimes 1)(W \otimes 1) \\
= & \left.\times\left(h \otimes W_{G}^{*}(\xi \otimes \eta)\right)\right\} \\
= & \left(1 \otimes W_{G}\right)(\pi \otimes i(\delta(a)) \otimes 1)\left(1 \otimes W_{G}^{*}\right)\left(1 \otimes W_{G}\right)(V \otimes 1 \otimes 1) \\
& \times(W \otimes i(\delta(a)))\left\{(V \otimes 1 \otimes 1)\left(1 \otimes W_{G}\right)(W \otimes 1)\right. \\
= & \left.\times\left(1 \otimes W_{G}^{*}\right)(h \otimes \xi \otimes \eta)\right\} \\
& \sim L .
\end{aligned}
$$

Again the approximations can be made arbitrarily accurate, so $Y$ satisfies the corepresentation identity.

To show that $(\pi, Y)$ is a covariant representation of $(A, G, \delta)$ we have to prove that

$$
\pi \otimes i(\delta(a))=Y(\pi(a) \otimes 1) Y^{*} \text { for } a \in A .
$$

Let $b \otimes \zeta$ be an elementary tensor in $A \odot\left(\mathscr{H} \otimes L^{2}(G)\right)$; then

$$
\begin{aligned}
\pi \otimes i(\delta(a)) Y(b \otimes \zeta) & =\pi \otimes i(\delta(a)) \pi \otimes i(\delta(b))(V \otimes 1(W \zeta)) \\
& =\pi \otimes i(\delta(a b))(V \otimes 1(W \zeta)) \\
& =Y(a b \otimes \zeta) \\
& =Y(\pi(a) \otimes 1)(b \otimes \zeta) .
\end{aligned}
$$

So $(\pi, Y)$ is a covariant pair. The consistency condition (c) also follows from an approximation. Note that

$$
Y(V \otimes 1(\zeta)) \sim Y\left(e_{\gamma} \otimes \zeta\right)=(\pi \otimes i)\left(\delta\left(e_{\gamma}\right)\right)(V \otimes 1)(W(\zeta)) .
$$

Because $\delta\left(e_{\gamma}\right)$ converges to 1 strictly in $M\left(A \otimes C_{r}^{*}(G)\right)$, and $\pi \otimes i$ is non-degenerate, the right-hand side converges to $V \otimes 1(W(\zeta))$, and this proves the first inequality in (c). To prove the second we evaluate both sides on a generator $a \otimes W^{*}(h \otimes y \cdot \eta)$ for $\mathscr{K}$, where $a \in A, \eta \in L^{2}(G)$, and $y \in C_{r}^{*}(G)$. We choose $\sum a_{j} \otimes z_{j}$ near $\delta(a)(1 \otimes y)$, and $\gamma$ such that $e_{\gamma} \otimes h \otimes \eta$ is close to $(V \otimes 1)(h \otimes \eta)$ and such that $\left\|a_{j} e_{\gamma}-a_{j}\right\|$ is small 
for all $j$. Then

$$
\begin{aligned}
\left(V^{*}\right. & \otimes 1) Y\left(a \otimes W^{*}(h \otimes y \cdot \eta)\right) \\
& =\left(V^{*} \otimes 1\right)(\pi \otimes i(\delta(a)))(V \otimes 1)(h \otimes y \cdot \eta) \\
& =\left(V^{*} \otimes 1\right)(\pi \otimes i(\delta(a))(1 \otimes y))(V \otimes 1)(h \otimes \eta) \\
& \sim\left(V^{*} \otimes 1\right)\left(\sum_{j}\left(\pi\left(a_{j}\right) \otimes z_{j}\right)\left(e_{\gamma} \otimes h \otimes \eta\right)\right) \\
& =\left(V^{*} \otimes 1\right)\left(\sum_{j} a_{j} e_{\gamma} \otimes h \otimes z_{j} \cdot \eta\right) \\
& =\sum_{j} \Phi\left(a_{j} e_{\gamma}\right) h \otimes z_{j} \cdot \eta \\
& \sim \sum_{j} \Phi\left(a_{j}\right) h \otimes z_{j} \cdot \eta \\
& =\sum_{j}(\Phi \otimes i)\left(a_{j} \otimes z_{j}\right)(h \otimes \eta) \\
& \sim \Phi \otimes i(\delta(a)(1 \otimes y))(h \otimes \eta) \\
& =\Phi \otimes i(\delta(a))(h \otimes y \cdot \eta) \\
& =W(\Phi(a) \otimes 1) W^{*}(h \otimes y \cdot \eta) \\
& =W\left(V^{*} \otimes 1\right)\left(a \otimes W^{*}(h \otimes y \cdot \eta)\right) .
\end{aligned}
$$

This proves (c) and it remains to verify (d).

Let $x \in \Phi(A)^{\prime}$ and $a \in A$. Then

$$
(x \otimes 1) W(\Phi(a) \otimes 1) W^{*} \in \mathscr{B}(\mathscr{H}) \bar{\otimes} v N(G),
$$

and the slice maps $S_{f}$, for $f \in A(G) \cong v N(G)_{*}$, may be applied to it. Since property (1) in the definition of a coaction implies that $S_{f}(\delta(a)) \in A$, the equivariance of $\Phi$ gives

$$
\begin{aligned}
& S_{f}\left((x \otimes 1) W(\Phi(a) \otimes 1) W^{*}\right) \\
& \quad=x S_{f}\left(W(\Phi(a) \otimes 1) W^{*}\right)=x \Phi\left(S_{f}(\delta(a))\right) \\
& \quad=\Phi\left(S_{f}(\delta(a))\right) x=S_{f}\left(W(\Phi(a) \otimes 1) W^{*}(x \otimes 1)\right) .
\end{aligned}
$$

Thus

$$
(x \otimes 1) W(\Phi(a) \otimes 1) W^{*}=W(\Phi(a) \otimes 1) W^{*}(x \otimes 1),
$$

and therefore

$$
W^{*}(x \otimes 1) W(\Phi(a) \otimes 1)=(\Phi(a) \otimes 1) W^{*}(x \otimes 1) W,
$$


so

$$
W^{*}(x \otimes 1) W \in(\Phi(A) \otimes 1)^{\prime} .
$$

Finally for $a$ in $A$ and $\zeta$ in $\mathscr{H} \otimes L^{2}(G)$, a direct computation gives the last part of $(\mathrm{d})$ :

$$
\begin{aligned}
Y(\rho & \left.\otimes i\left(W^{*}(x \otimes 1) W\right)(a \otimes \zeta)\right) \\
& =Y\left(a \otimes\left(W^{*}(x \otimes 1) W(\zeta)\right)\right) \\
& =(\pi \otimes i)(\delta(a))\{(\rho(x) \otimes 1)(V \otimes 1) W(\zeta)\} \quad \text { by }(\mathrm{d}) \\
& =(\rho(x) \otimes 1)(\pi \otimes i(\delta(a))((V \otimes 1) W(\zeta))) \\
& =(\rho(x) \otimes 1) Y(a \otimes \zeta)
\end{aligned}
$$

for all $a \in A$ and $\zeta \in \mathscr{H} \otimes L^{2}(G)$. This completes the proof of Theorem 4.1.

REMARK 4.4. When the completely positive map is scalar-valuedi.e., a positive functional-Stinespring's theorem reduces to the Gelfand-Naimark-Segal construction, and the equivariant version (Lemma 3.1) reduces to the well-known construction of a covariant representation of $(A, G, \alpha)$ from an invariant functional. It seems reasonable to say that a positive functional $\omega \in A^{*}$ is invariant under a coaction $\delta$ if

$$
L S_{\omega}(\delta(a))=\omega(a) 1 \quad \text { in } M\left(C_{r}^{*}(G)\right) \text { for all } a \in A \text {. }
$$

For such a functional, Theorem 4.1 gives a covariant representation $\left(\pi_{\omega}, Y_{\omega}\right)$ of $(A, G, \delta)$, where $\pi_{\omega}$ is the usual GNS-representation of $A$ associated to $\omega$.

In this case, the representation $\mu$ of $A(G)$ corresponding to the corepresentation $Y_{\omega}$ is given on the dense subspace $A$ of $\mathscr{H}_{\omega}$ by $\mu(f)(a)$ $=R S_{f}(\delta(a))$ for $f \in A(G)$. To see this, choose $\xi, \eta \in L^{2}(G)$ such that $(f, y)=\langle y \xi, \eta\rangle$ for $y \in C_{r}^{*}(G)$, and suppose for simplicity that $A$ has an identity. Then for $a, b \in A$ we have

$$
\begin{aligned}
\left\langle S_{f}\left(Y_{\omega}\right)(a), b\right\rangle_{\omega} & =\left\langle Y_{\omega}(a \otimes \xi), b \otimes \eta\right\rangle \\
& =\left\langle\pi_{\omega} \otimes i(\delta(a))(1 \otimes \xi), b \otimes \eta\right\rangle \\
& =\left\langle S_{f}\left(\pi_{\omega} \otimes i(\delta(a))\right)(1), b\right\rangle_{\omega} \\
& =\left\langle\pi_{\omega}\left(S_{f}(\delta(a))\right)(1), b\right\rangle_{\omega}=\left\langle S_{f}(\delta(a)), b\right\rangle_{\omega},
\end{aligned}
$$

as required. It is interesting to note that, while it is quite easy to verify directly that $f \mapsto S_{f}(\delta(a))$ is a well-defined homomorphism, it does not appear to be so obvious that $\mu$ is $*$-preserving. Thus the GNS construction may not be significantly easier than our Stinespring theorem. 
THeOREM 4.5. Let $\delta$ be a coaction of a group $G$ on a $C^{*}$-algebra $A$, and let $W$ be a corepresentation of $G$ on a Hilbert space $\mathscr{H}$. If $\Phi$ is an equivariant symmetric completely bounded linear operator from $A$ into $\mathscr{B}(\mathscr{H})$, then there are equivariant completely positive linear operators $\Phi_{1}$ and $\Phi_{2}$ from $A$ into $\mathscr{B}(\mathscr{H})$ such that $\Phi=\Phi_{1}-\Phi_{2}$ and $\|\Phi\|_{c b}=$ $\left\|\Phi_{1}(1)+\Phi_{2}(1)\right\|$.

This result is the coaction version of Lemma 3.2, and the proof has the same overall structure though the averaging is more complicated because of the coaction. By the usual Wittstock theorem [25] completely positive maps $\tau_{1}$ and $\tau_{2}$ can be chosen satisfying the conclusions except for the equivariance. It will therefore be enough for us to construct from each completely bounded map $\tau$ an equivariant completely bounded map $m(\tau)$ in such a way that $m(\tau)$ depends linearly on $\tau, m(\tau)$ is completely positive if $\tau$ is and $m(\tau)=\tau$ if $\tau$ is already equivariant. The idea is to average $\tau$ using an invariant mean $m$ on the von Neumann algebra $v N(G)=\lambda(G)^{\prime \prime}$, just as an invariant mean on $L^{\infty}(G)$ can be used to average bounded functions on an amenable group $G$.

In order to write down the appropriate invariance condition this mean must satisfy, we shall need to extend the usual notion of slice map to non-normal functionals on a von Neumann algebra. We shall deal with the right slice maps $R S_{f}$-obviously we can define left slice maps $L S_{f}$ with similar properties. Recall that $\bar{\otimes}$ denotes the von Neumann algebra spatial tensor product of two von Neumann algebras.

LemMa 4.6. Suppose $M, N$ are von Neumann algebras acting on $\mathscr{H}, \mathscr{L}$ respectively, and $f \in N^{*}$. For each $T \in \mathscr{B}(\mathscr{H}) \bar{\otimes} N$ we define an operator $R S_{f}(T) \in \mathscr{B}(\mathscr{H})$ by

$$
\left(R S_{f}(T) h \mid k\right)=\left\langle f, L S_{\omega_{h, k}}(T)\right\rangle \quad \text { for } h, k \in \mathscr{H},
$$

where $L S_{\omega_{h, k}}$ denotes the usual, spatially defined, slice map corresponding to the normal functional $\omega_{h, k}: Q \mapsto(Q h \mid k)$ on $\mathscr{B}(\mathscr{H})$. Then:

(1) $R S_{f}$ is a bounded linear map of norm $\leq\|f\|$.

(2) If $f \geq 0$ in $N^{*}$ and $T \geq 0$ in $M \bar{\otimes} N$, then $R S_{f}(T) \geq 0$ in $\mathscr{B}(\mathscr{H})$.

(3) If $U \in \mathscr{B}(\mathscr{H})$ and $Q \in N$, then

$$
\begin{gathered}
U\left(R S_{f}(T)\right)=R S_{f}((U \otimes 1) T), \quad R S_{f}(T) U=R S_{f}(T(U \otimes 1)), \\
R S_{Q \cdot f}(T)=R S_{f}(T(1 \otimes Q)), \quad R S_{f \cdot Q}(T)=R S_{f}((1 \otimes Q) T),
\end{gathered}
$$

where, for example, $Q \cdot f$ is the functional $T \mapsto f(T Q)$ on $N$.

(4) If $T \in M \bar{\otimes} N$, then $R S_{f}(T) \in M$. 
Proof. We notice that the right-hand side of $(*)$ is a bounded sesquilinear form in $h, k$ of norm $\leq\|f\|\|T\|$, so $R S_{f}$ is certainly a bounded linear map of $\mathscr{B}(\mathscr{H}) \bar{\otimes} N$ to $\mathscr{B}(\mathscr{H})$ of norm $\leq\|f\|$. If $T \geq 0$ and $h \in \mathscr{H}$, then $L S_{\omega_{h, h}}(T) \geq 0$ in $N$. It follows that

$$
\left(R S_{f}(T) h \mid h\right)=\left\langle f, L S_{\omega_{h, h}}(T)\right\rangle \geq 0 \text { for all } h \in H,
$$

so $R S_{f}(T) \geq 0$ in $\mathscr{B}(\mathscr{H})$ and (2) holds. If $U \in \mathscr{B}(\mathscr{H})$, then

$$
\begin{aligned}
& \left(U R S_{f}(T) h \mid k\right)=\left\langle f, L S_{\omega_{h U^{*} k}}(T)\right\rangle \\
& \quad=\left\langle f, L S_{\omega_{h, h}}((U \otimes 1) T)\right\rangle=\left(R S_{f}((U \otimes 1) T) h \mid k\right) ;
\end{aligned}
$$

the other parts of (3) follow similarly from the analogous properties of the usual slice maps. Finally, suppose $T \in M \bar{\otimes} N$ and $Q \in M^{\prime}$. Then $Q \otimes 1 \in(M \bar{\otimes} N)^{\prime}$, and by (3) we have

$$
Q\left(R S_{f}(T)\right)=R S_{f}((Q \otimes 1) T)=R S_{f}(T(Q \otimes 1))=R S_{f}(T) Q
$$

thus $R S_{f}(T) \in\left(M^{\prime}\right)^{\prime}=M$. This completes the proof.

Recall that the comultiplication $\delta_{G}$ extends to a homomorphism $\delta_{G}: v N(G) \rightarrow v N(G) \bar{\otimes} v N(G)$; to see this, just observe that $\delta_{G}$ is given spatially by

$$
\delta_{G}(T)=W_{G}(T \otimes 1) W_{G}^{*}
$$

Proposition 4.7. There is a positive functional $m$ on $v N(G)$ such that

$$
L S_{m}\left(\delta_{G}(T)\right)=\langle m, T\rangle 1 \quad \text { for } T \in v N(G) .
$$

Proof. Choose a decreasing net $\left\{K_{\alpha}\right\}$ of compact neighborhoods of $e$ with $\bigcap_{\alpha} K_{\alpha}=\{e\}$, and let $\xi_{\alpha}=\mu\left(K_{\alpha}\right)^{-1 / 2} \chi_{K_{\varepsilon}}$, so that each $\xi_{\alpha}$ is a unit vector in $L^{2}(G)$. Let $f_{\alpha} \in A(G)$ be the functions

$$
f_{\alpha}(s)=\int \xi_{\alpha}\left(s^{-1} t\right) \overline{\xi_{\alpha}(t)} d t,
$$

so that for $z \in L^{1}(G)$ we have $\left\langle z, f_{\alpha}\right\rangle=\left(z * \xi_{\alpha} \mid \xi_{\alpha}\right)$. Then by Hölder's inequality

$$
\left|f_{\alpha}(s)\right| \leq \int\left|\xi_{\alpha}\left(s^{-1} t\right)\right|\left|\xi_{\alpha}(t)\right| d t \leq\left\|\lambda_{s}^{-1}\left(\xi_{\alpha}\right)\right\|_{2}\left\|\xi_{\alpha}\right\|_{2}=1,
$$

and we have

$$
\left\|f_{\alpha}\right\|_{A(G)} \leq 1
$$

in other words, the net $\left\{f_{\alpha}\right\}$ is bounded in $A(G)=v N(G)_{*} \subset v N(G)^{*}$. We can therefore suppose by passing to a subnet that $\left\{f_{\alpha}\right\}$ converges 
in the weak $*$-topology-to $m$, say. We claim that this limit has the required property.

We have to prove that, for any $T \in v N(G)$ and $\xi, \eta \in L^{2}(G)$,

$$
\left(L S_{m}\left(\delta_{G}(T)\right) \xi \mid \eta\right)=\langle m, T\rangle(\xi \mid \eta) .
$$

Let $g \in A(G)$ be given by

$$
g(s)=\int \xi\left(s^{-1} t\right) \overline{\eta(t)} d t .
$$

Then by definition of the left slice map (Lemma 4.6), the left-hand side of (1) is

$$
\begin{aligned}
\left\langle m, R S_{g}\left(\delta_{G}(T)\right)\right\rangle & =\lim _{\alpha}\left(R S_{g}\left(\delta_{G}(T)\right) \xi_{\alpha} \mid \xi_{\alpha}\right) \\
& =\lim _{\alpha}\left(\delta_{G}(T)\left(\xi_{\alpha} \otimes \xi\right) \mid \xi_{\alpha} \otimes \eta\right) \\
& =\lim _{\alpha}\left((T \otimes 1) W_{G}^{*}\left(\xi_{\alpha} \otimes \xi\right) \mid W_{G}^{*}\left(\xi_{\alpha} \otimes \eta\right)\right) .
\end{aligned}
$$

Now

$$
\begin{aligned}
\left\|W_{G}^{*}\left(\xi_{\alpha} \otimes \xi\right)-\xi_{\alpha} \otimes \xi\right\|^{2} & =\iint\left|W_{G}^{*}\left(\xi_{\alpha} \otimes \xi\right)(r, s)-\xi_{\alpha}(r) \xi(s)\right|^{2} d r d s \\
& =\iint\left|\xi_{\alpha}(r)\right|^{2}|\xi(r s)-\xi(s)|^{2} d s d r \\
& =\int\left|\xi_{\alpha}(r)\right|^{2}\left\|\lambda_{r}^{-1} \xi-\xi\right\|_{2}^{2} d r .
\end{aligned}
$$

The continuity of the left regular representation $\lambda$ implies that $\left\|\lambda_{r}^{-1} \xi-\xi\right\|_{2}^{2} \rightarrow 0$ as $r \rightarrow e$, and $\operatorname{supp} \xi_{\alpha}=K_{\alpha}$ is shrinking to $e$, so

$$
\left\|W_{G}^{*}\left(\xi_{\alpha} \otimes \xi\right)-\xi_{\alpha} \otimes \xi\right\|^{2} \rightarrow 0 \quad \text { as } \alpha \text { increases. }
$$

Of course, we have a similar convergence in the $\xi_{\alpha} \otimes \eta$ variable, so

$$
\left|\left((T \otimes 1) W_{G}^{*}\left(\xi_{\alpha} \otimes \xi\right) \mid W_{G}^{*}\left(\xi_{\alpha} \otimes \eta\right)\right)-\left(T \xi_{\alpha} \otimes \xi \mid \xi_{\alpha} \otimes \eta\right)\right| \rightarrow 0 .
$$

Thus

$$
\begin{aligned}
\left\langle m, R S_{g}\left(\delta_{G}(T)\right)\right\rangle & =\lim _{\alpha}\left((T \otimes 1) W_{G}^{*}\left(\xi_{\alpha} \otimes \xi\right) \mid W_{G}^{*}\left(\xi_{\alpha} \otimes \eta\right)\right) \\
& =\lim _{\alpha}\left(T \xi_{\alpha} \otimes \xi \mid \xi_{\alpha} \otimes \eta\right) \\
& =\langle m, T\rangle(\xi \mid \eta),
\end{aligned}
$$

and $m$ has the required invariance property. The mean $m$ is obviously positive.

REMARK 4.8. (1) Notice that the $f_{\alpha}$ constructed in the proof also satisfy

$$
f_{\alpha}(0)=\int \xi_{\alpha}(t) \overline{\xi_{\alpha}(t)} d t=\left(\xi_{\alpha} \mid \xi_{\alpha}\right)=1,
$$


and

$$
f_{\alpha}(s)=0 \quad \text { unless } s \in K_{\alpha} K_{\alpha}^{-1} \text {, }
$$

so that $f_{\alpha} \rightarrow \chi_{e}$ pointwise. If $G$ is second countable, then we can work with sequences throughout, and the dominated convergence theorem implies that for $\mu \in M(G)$

$$
\langle m, \mu\rangle=\lim _{n}\left\langle f_{n}, \mu\right\rangle=\lim _{n} \int f_{n}(s) d \mu(s)=\mu(\{e\}) .
$$

This should help give some intuitive feeling for the "mean" $m$.

(2) The functional $m$ is also a topologically invariant mean on $\hat{G}$ in the sense of Granirer [9]. To see this, let $V \in v N(G)$ and suppose $u \in A(G)$ satisfies $u \geq 0$ (as a functional on $v N(G)$ ) and $u(e)=1$. Then

$$
\langle m, u \cdot V\rangle=\lim _{\alpha}\left\langle f_{\alpha}, u \cdot V\right\rangle=\lim _{\alpha}\left\langle u f_{\alpha}, V\right\rangle,
$$

by definition of the module action of $A(G)$ on $v N(G)$. But

$$
\left\|u f_{\alpha}-f_{\alpha}\right\|_{A(G)} \rightarrow 0 \quad \text { since } u(e)=1 ;
$$

to see this, note we can approximate $u$ in $A(G)$ by a function which is identically 1 near $e,[7,4.11]$, and that the supports of the $f_{\alpha}$ are shrinking to $\{e\}$. Thus

$$
\left|\left\langle u f_{\alpha}, V\right\rangle-\left\langle f_{\alpha}, V\right\rangle\right| \rightarrow 0 \text { as } \alpha \text { increases, }
$$

and therefore

$$
\langle m, u \cdot V\rangle=\lim _{\alpha}\left\langle f_{\alpha}, V\right\rangle=\langle m, V\rangle .
$$

(3) After we had found this proof of Proposition 4.7 we discovered the result had already been obtained by Enock and Schwartz [6, Proposition 2.11]. They deduce it from a general discussion of amenability of Kac algebras [6, Théorème 2.4]; in their terminology, our $m$ is a right invariant mean on the Kac algebra $K S(G)$. We have preferred to retain this self-contained proof, but we refer to [6] for some interesting applications.

We are now in a position to define our averaged version of a completely bounded map $\tau: A \rightarrow \mathscr{B}(\mathscr{H})$ (such that $\tau(1)=1$ ). There is a unique strictly continuous extension of $\tau \otimes i$ to a map of $M\left(A \otimes C_{r}^{*}(G)\right)$ into $\mathscr{B}\left(\mathscr{H} \otimes L^{2}(G)\right)$, and we can define

$$
m(\tau)(a)=R S_{m}\left(W^{*}(\tau \otimes i(\delta(a))) W\right) .
$$

Notice right away that if $\tau$ is already equivariant, then

$$
S_{f}(\tau \otimes i(\delta(a)))=\tau\left(S_{f}(\delta(a))\right)=S_{f}\left(W(\tau(a) \otimes 1) W^{*}\right) \quad \text { for all } f \in A^{*}
$$


which implies

$$
\tau \otimes i(\delta(a))=W(\tau(a) \otimes 1) W^{*},
$$

and hence that

$$
m(\tau)(a)=R S_{m}(\tau(a) \otimes 1)=\langle m, 1\rangle \tau(a)=\tau(a)
$$

(taking $T=\delta_{e}$ in the invariance condition forces $\langle m, 1\rangle=1$ ). The map

$$
a \mapsto W^{*}(\tau \otimes i(\delta(a))) W: A \rightarrow \mathscr{B}(\mathscr{H}) \bar{\otimes} v N(G)
$$

is completely bounded, so to verify that $m(\tau)$ is always completely bounded we just need the following lemma.

LEMMA 4.9. Suppose $T: A \rightarrow \mathscr{B}(\mathscr{H}) \bar{\otimes} N$ is completely bounded and $f \in N^{*}$. Then $R S_{f} \circ T$ is completely bounded; it is also completely positive if $T$ is and if $f \geq 0$.

Proof. Suppose $\left(a_{i j}\right) \in M_{n}(A)$. Then

$$
\left(\left(R S_{f} \circ T\right) \otimes i_{n}\right)\left(a_{i j}\right)=\left(R S_{f} \circ T\left(a_{i j}\right)\right)=R S_{f}^{n}\left(T\left(a_{i j}\right)\right),
$$

where $R S_{f}^{n}$ denotes the slice map from $\mathscr{B}(\mathscr{H}) \bar{\otimes} N \otimes M_{n}$ to $\mathscr{B}(\mathscr{H}) \otimes M_{n}$. (To see this last equality, let $\left\{e_{k}\right\}$ be the usual basis for $\mathbf{C}^{n}$ and apply the vector functional $R \mapsto\left(R\left(h \otimes e_{k}\right) \mid k \otimes e_{l}\right)$ to both sides.) Thus

$$
\begin{aligned}
\left\|\left(\left(R S_{f} \circ T\right) \otimes i_{n}\right)\left(a_{i j}\right)\right\| & \leq\left\|R S_{f}^{n}\right\|\left\|T \otimes i_{n}\right\|\left\|\left(a_{i j}\right)\right\| \\
& \leq\|f\|\left\|\left(a_{i j}\right)\right\|\|T\|_{c b},
\end{aligned}
$$

and $R S_{f} \circ T$ is completely bounded. If $f \geq 0, T$ is completely positive and $\left(a_{i j}\right) \geq 0$ in $M_{n}(A)$, then $\left(T\left(a_{i j}\right)\right) \geq 0$ in $\mathscr{B}(\mathscr{H}) \otimes M_{n}$. The positivity of $\left(\left(R S_{f} \circ T\right) \otimes i\right)\left(a_{i j}\right)$ now follows from (1) and the positivity of $R S_{f}^{n}$ (Lemma 4.6). This proves the lemma.

To prove Theorem 4.5 it remains only to verify that $m(\tau)$ is $\delta-$ Ad $W$ equivariant. So we have to prove that

$$
m(\tau)\left(R S_{g}(\delta(a))\right)=R S_{g}\left(W(m(\tau)(a) \otimes 1) W^{*}\right)
$$

for $a \in A$ and $g \in A(G)$, or, equivalently, that

$$
\begin{aligned}
& R S_{m}\left(W^{*}\left(\tau \otimes i\left(\delta\left(R S_{g}(\delta(a))\right)\right)\right) W\right) \\
& \quad=R S_{g}\left(W\left(R S_{m}\left(W^{*}(\tau \otimes i(\delta(a)) W)\right) W^{*}\right) .\right.
\end{aligned}
$$

We're going to want to juggle these slice maps around, and this will require a little care since $R S_{m}$ is not the usual spatially-defined slice map. We introduce the outer slice map

$$
O S_{\omega \otimes g}=L S_{\omega} \circ R S_{g}=R S_{g} \circ L S_{\omega}: L \bar{\otimes} N \bar{\otimes} M \rightarrow N
$$


for $\omega \in L_{*}, g \in M_{*}$, and the middle slice map

$$
M S_{f}: L \bar{\otimes} N \bar{\otimes} M \rightarrow L \bar{\otimes} M,
$$

for $f \in N^{*}$, which is defined by

$$
\left(M S_{f}(T)(h \otimes \zeta) \mid k \otimes \eta\right)=\left\langle f, O S_{\omega_{h, k} \otimes \omega_{\zeta, \eta}}(T)\right\rangle
$$

for $h, k \in \mathscr{H}(L)$ and $\zeta, \eta \in \mathscr{H}(M)$. Notice that, modulo the flip $\sigma: L \bar{\otimes} N \bar{\otimes} \rightarrow L \bar{\otimes} M \bar{\otimes} N, M S_{f}$ is just the right slice map $R S_{f}$ defined in Lemma 4.6, and therefore has all the same properties. We introduced $M S_{f}$ because

$$
R S_{f} \circ R S_{g}=R S_{g} \circ M S_{f} \text { for } f \in N^{*}, g \in M_{*} .
$$

To see this, note that if $g=\omega_{\zeta, \eta}$, then

$$
\begin{aligned}
\left(R S_{f}\right. & \left.\circ R S_{g}(T) h \mid k\right)=\left\langle f, L S_{\omega_{h, k}} \circ R S_{g}(T)\right\rangle \\
& =\left\langle f, O S_{\omega_{h, k} \otimes g}(T)\right\rangle=\left(M S_{f}(T)(h \otimes \zeta) \mid k \otimes \eta\right) \\
& =\left(R S_{g}\left(M S_{f}(T)\right) h \mid k\right) .
\end{aligned}
$$

With these conventions, the left-hand side of (2) becomes

$$
\begin{array}{r}
R S_{m}\left(W^{*}\left(\tau \otimes i\left(R S_{g}(\delta \otimes i(\delta(a)))\right)\right) W\right) \\
=R S_{m} \circ R S_{g}\left(\left(W^{*} \otimes 1\right)\left(\tau \otimes i \otimes i\left(i \otimes \delta_{G}(\delta(a))\right)\right)(W \otimes 1)\right) \\
\text { using the coaction identity } \\
=R S_{g} \circ M S_{m}\left(\left(W^{*} \otimes 1\right) i \otimes \delta_{G}(\tau \otimes i(\delta(a)))(W \otimes 1)\right) \text { using }(3) \\
=R S_{g} \circ M S_{m}\left(\left(W^{*} \otimes 1\right)\left(1 \otimes W_{G}\right)((\tau \otimes i(\delta(a))) \otimes 1)\right. \\
=R S_{g} \circ M S_{m}\left((W \otimes 1)^{\sigma}\left(1 \otimes W_{G}\right)\left(W^{*} \otimes 1\right)\right. \\
\left.\times((\tau \otimes i(\delta(a))) \otimes 1)(W \otimes 1)\left(1 \otimes W_{G}^{*}\right)(W \otimes 1)\right) \\
\quad \text { using the fact that } W \text { is a corepresentation } \\
=R S_{g}\left(W M S_{m}\left(\left(1 \otimes W_{G}\right)\left(\left(W^{*} \tau \otimes i(\delta(a)) W\right) \otimes 1\right)\left(1 \otimes W_{G}^{*}\right)\right) W^{*}\right)
\end{array}
$$

by Lemma 4.6. Let $T \in \mathscr{B}(\mathscr{H}) \bar{\otimes} v N(G), h, k \in \mathscr{H}, \eta_{i} \in L^{2}(G)$ and define $g \in A(G)$ by

$$
g(s)=\int \eta_{1}\left(s^{-1} t\right) \overline{\eta_{2}(t)} d t
$$


Then

$$
\begin{aligned}
\left(M S_{m}\right. & \left.\left(\left(1 \otimes W_{G}\right)(T \otimes 1)\left(1 \otimes W_{G}^{*}\right)\right)\left(h \otimes \eta_{1}\right) \mid k \otimes \eta_{2}\right) \\
& =\left(M S_{m}\left(i \otimes \delta_{G}(T)\right)\left(h \otimes \eta_{1}\right) \mid k \otimes \eta_{2}\right) \\
& =\left\langle m, O S_{\omega_{h, k} \otimes g}\left(i \otimes \delta_{G}(T)\right)\right\rangle \\
& =\left\langle m, R S_{g}\left(L S_{\omega_{h, k}}\left(i \otimes \delta_{G}(T)\right)\right)\right\rangle \\
& =\left\langle m, R S_{g}\left(\delta_{G}\left(L S_{\omega_{h, k}}(T)\right)\right)\right\rangle \\
& =\left(L S_{m}\left(\delta_{G}\left(L S_{\omega_{h, k}}(T)\right)\right) \eta_{1} \mid \eta_{2}\right) \\
& =\left\langle m, L S_{\omega_{h, k}}(T)\right\rangle\left(\eta_{1} \mid \eta_{2}\right) \quad \text { since } m \text { is invariant } \\
& =\left(R S_{m}(T) h \mid k\right)\left(\eta_{1} \mid \eta_{2}\right) \\
& =\left(\left(R S_{m}(T) \otimes 1\right)\left(h \otimes \eta_{1}\right) \mid k \otimes \eta_{2}\right) .
\end{aligned}
$$

Applying this with $T=\left(W^{*}(\tau \otimes i(\delta(a))) W\right)$ gives immediately that the left-hand side of $(2)$ is equal to

$$
R S_{g}\left(W\left(R S_{m}\left(W^{*}(\tau \otimes i(\delta(a))) W\right) \otimes 1\right) W^{*}\right),
$$

which is just the right-hand side of (2).

This completes the proof of Theorem 4.5.

The direct sum of two representations of a group $G$ is a representation; the following lemma is the analogous result for corepresentations.

LEMMA 4.10. Let $Y_{1}$ and $Y_{2}$ be corepresentations of a group $G$ on Hilbert spaces $\mathscr{H}_{1}$ and $\mathscr{H}_{2}$ and let $i_{j}: \mathscr{H}_{j} \rightarrow \mathscr{H}=\mathscr{H}_{1} \oplus \mathscr{H}_{2}$ denote the natural inclusions. Let

$$
u:\left(\mathscr{H}_{1} \otimes L^{2}(G)\right) \oplus\left(\mathscr{H}_{2} \otimes L^{2}(G)\right) \rightarrow \mathscr{H} \otimes L^{2}(G)
$$

be defined by

$$
u(\xi, \eta)=\left(i_{1} \otimes 1\right)(\xi)+\left(i_{2} \otimes 1\right)(\eta) .
$$

Then $u$ is a unitary operator and

$$
Y=u\left(\begin{array}{cc}
Y_{1} & 0 \\
0 & Y_{2}
\end{array}\right) u^{*}
$$

is a corepresentation of $G$ on $\mathscr{H}$.

Proof. Clearly $u$ is isometric and has inverse given by

$$
u^{-1}(k)=\left(p_{1} \otimes 1\right)(k) \oplus\left(p_{2} \otimes 1\right)(k),
$$

where $p_{j}$ is the projection of $\mathscr{H}_{1} \oplus \mathscr{H}_{2}$ onto $\mathscr{H}_{j}$. If

$$
\begin{aligned}
V:\left(\mathscr{H} \otimes L^{2}(G) \otimes L^{2}(G)\right) & \oplus\left(\mathscr{H} \otimes L^{2}(G) \otimes L^{2}(G)\right) \\
& \rightarrow(\mathscr{H} \oplus \mathscr{H}) \otimes L^{2}(G) \otimes L^{2}(G)
\end{aligned}
$$


is defined in a similar fashion to $u$, then $Y \otimes 1$ can be described in terms of $Y_{j} \otimes 1$ by

$$
Y \otimes 1=V\left(\begin{array}{cc}
Y_{1} \otimes 1 & 0 \\
0 & Y_{2} \otimes 1
\end{array}\right) V^{*} .
$$

Recall that $\sigma: L^{2}(G) \otimes L^{2}(G) \rightarrow L^{2}(G) \otimes L^{2}(G)$ is the flip $\sigma(\xi \otimes \eta)=$ $\eta \otimes \xi$, and that $\left(Y_{j} \otimes 1\right)^{\sigma}=(1 \otimes \sigma)\left(Y_{j} \otimes 1\right)(1 \otimes \sigma)$ and similarly for $Y$. Now

$$
(1 \otimes \sigma) V((1 \otimes \sigma) \oplus(1 \otimes \sigma))=V
$$

so that

$$
\begin{aligned}
& (Y \otimes 1)(Y \otimes 1)^{\sigma} \\
& =V\left(\begin{array}{cc}
Y_{1} \otimes 1 & 0 \\
0 & Y_{2} \otimes 1
\end{array}\right) V^{*} V\left(\begin{array}{cc}
\left(Y_{1} \otimes 1\right)^{\sigma} & 0 \\
0 & \left(Y_{2} \otimes 1\right)^{\sigma}
\end{array}\right) V^{*} \\
& =V\left(\begin{array}{cc}
\left(Y_{1} \otimes 1\right)\left(Y_{1} \otimes 1\right)^{\sigma} & 0 \\
0 & \left(Y_{2} \otimes 1\right)\left(Y_{2} \otimes 1\right)^{\sigma}
\end{array}\right) V^{*} \\
& =V\left(\begin{array}{cc}
1 \otimes W_{G} & 0 \\
0 & 1 \otimes W_{G}
\end{array}\right)\left(\begin{array}{cc}
Y_{1} \otimes 1 & 0 \\
0 & Y_{2} \otimes 1
\end{array}\right) \\
& \times\left(\begin{array}{cc}
1 \otimes W_{G}^{*} & 0 \\
0 & 1 \otimes W_{G}^{*}
\end{array}\right) V^{*} \\
& =\left\{V\left(\begin{array}{cc}
1 \otimes W_{G} & 0 \\
0 & 1 \otimes W_{G}
\end{array}\right) V^{*}\right\} \\
& \times(Y \otimes 1)\left\{V\left(\begin{array}{cc}
1 \otimes W_{G} & 0 \\
0 & 1 \otimes W_{G}
\end{array}\right) V^{*}\right\} \\
& =\left(1 \otimes W_{G}\right)(Y \otimes 1)\left(1 \otimes W_{G}\right) .
\end{aligned}
$$

Hence $Y$ is a corepresentation.

THEOREM 4.11. Let $\delta$ be a non-degenerate coaction of a locally compact group $G$ on a $C^{*}$-algebra $A$, and let $W$ be a corepresentation of $G$ on a Hilbert space $\mathscr{H}$. If $\Phi$ is an equivariant completely bounded linear operator from $A$ into $\mathscr{B}(\mathscr{H})$, then there are

(1) a covariant representation $(\pi, Y)$ of $(A, G, \delta)$ on a Hilbert space $\mathscr{K}$, and

(2) continuous linear operators $V: \mathscr{H} \rightarrow \mathscr{K}$, and $T: \mathscr{K} \rightarrow \mathscr{H}$ such that

(a) $\Phi(a)=T \pi(a) V$,

(b) $(V \otimes 1) W=Y(V \otimes 1)$ and $(T \otimes 1) Y=W(T \otimes 1)$, and

(c) $\|\Phi\|_{c b}=\|T\|\|V\|$. 
Proof. The proof is basically similar to the proof of Lemma 3.2. First we reduce to the case where $\Phi$ is symmetric. To do this, let $\Phi: A \rightarrow \mathscr{B}(\mathscr{H} \oplus \mathscr{H})$ be defined by

$$
\Phi(a)=\left(\begin{array}{cc}
0 & \Phi(a) \\
\Phi^{*}(a) & 0
\end{array}\right) ;
$$

then $\Phi$ is symmetric and completely bounded with $\|\Phi\|_{c b}=\|\Phi\|_{c b}$. In the notation of the lemma with $\mathscr{H}=\mathscr{H}_{1}=\mathscr{H}_{2}$ we set

$$
\underline{W}=u\left(\begin{array}{cc}
W & 0 \\
0 & W
\end{array}\right) u^{*} \in \mathscr{B}\left((\mathscr{H} \oplus \mathscr{H}) \otimes L^{2}(G)\right) .
$$

It is straightforward to verify that

$$
u\left(\begin{array}{cc}
0 & \Phi(a) \otimes 1 \\
\Phi^{*}(a) \otimes 1 & 0
\end{array}\right) u^{*}=\Phi(a) \otimes 1,
$$

and that, for all $f \in A(G)$ and all $Q, R, S, T \in \mathscr{B}\left(\mathscr{H} \otimes L^{2}(G)\right)$,

$$
S_{f}\left(u\left(\begin{array}{ll}
Q & R \\
S & T
\end{array}\right) u^{*}\right)=\left(\begin{array}{ll}
S_{f}(Q) & S_{f}(R) \\
S_{f}(S) & S_{f}(T)
\end{array}\right)
$$

as operators on $\mathscr{H} \oplus \mathscr{H}$. The identity $S_{f}(c)^{*}=S_{f^{*}}\left(c^{*}\right)$ implies that $\Phi^{*}$ is also $\delta-\operatorname{Ad} W$ equivariant. Putting all this together gives

$$
\begin{aligned}
\underline{\Phi}\left(S_{f}(\delta(a))\right. & =\left(\begin{array}{cc}
0 & S_{f}\left(W(\Phi(a) \otimes 1) W^{*}\right) \\
S_{f}\left(W\left(\Phi^{*}(a) \otimes 1\right) W^{*}\right) & 0
\end{array}\right) \\
& =S_{f}\left(u\left(\begin{array}{cc}
W & 0 \\
0 & W
\end{array}\right) u^{*} u\left(\begin{array}{cc}
0 & \Phi(a) \otimes 1 \\
\Phi^{*}(a) \otimes 1 & 0
\end{array}\right) u^{*} u\right. \\
& =S_{f}\left(\underline{W}(\underline{\Phi}(a) \otimes 1) \underline{W}^{*}\right)
\end{aligned}
$$

so that $\Phi$ is $(\delta-\operatorname{Ad} \underline{W})$-equivariant. If $\pi, Y, \underline{T}, \underline{V}$ can be found satisfying conditions $(a, b, c)$ appropriate to $\Phi$ and $\underline{W}$, then let $T=$ $p_{1} \underline{T}$ and $V=\underline{V} i_{2}$, where $p_{1}$ is the projection of $\mathscr{H} \oplus \mathscr{H}$ onto the first coordinate $\mathscr{H}$ and $i_{2}$ is the embedding of $\mathscr{H}$ into $\mathscr{H} \oplus \mathscr{H}$ onto the second coordinate. Conditions (a) and (c) are easily checked, and (b) follows from the analogous properties of $\underline{T}$ and $\underline{V}$, and the observation that

$$
\left(i_{2} \otimes 1\right) W=\underline{W}\left(i_{2} \otimes 1\right) \quad \text { and }\left(p_{1} \otimes 1\right) \underline{W}=W\left(p_{2} \otimes 1\right) .
$$

Therefore we shall suppose that $\Phi$ itself is symmetric. Apply Theorem 4.5 and Theorem 4.1 in the standard way one after the other (see 
the use of 2.1 in the proof of Theorem 2.2). Thus there are covariant representations $\left(\pi_{j}, Y_{j}\right)$ of $(A, G, \delta)$ on Hilbert spaces $\mathscr{K}_{j}$ and bounded linear operators $V_{j}: \mathscr{H} \rightarrow \mathscr{K}_{j}(j=1,2)$ such that

$$
\Phi(a)=V_{1}^{*} \pi_{1}(a) V_{1}-V_{2}^{*} \pi_{2}(a) V_{2}
$$

for all $a \in A,\|\Phi\|_{c b}=\left\|V_{1}^{*} V_{1}+V_{2}^{*} V_{2}\right\|$, and $V, Y_{j}, W$ satisfy the intertwining condition (c) of Theorem 4.1. We now let $\mathscr{K}=\mathscr{K}_{1} \oplus \mathscr{K}_{2}$, $\pi=\pi_{1} \oplus \pi_{2}$,

$$
T=\left(V_{1}^{*} V_{2}^{*}\right), \quad V=\left(\begin{array}{c}
V_{1} \\
-V_{2}
\end{array}\right), \quad \text { and } \quad Y=u\left(\begin{array}{cc}
Y_{1} & 0 \\
0 & Y_{2}
\end{array}\right) u^{*},
$$

where $u$ is as in Lemma 4.10. Conclusions (a) and (b) are checked as in Theorem 3.3. The covariance of $(\pi, Y)$ and the intertwining property (b) follow directly from the corresponding properties of $Y_{j}$ $(j=1,2)$. Property (c) follows from $\|T\|=\|V\|=\left\|V_{1}^{*} V_{1}+V_{2}^{*} V_{2}\right\|^{1 / 2}$ and $\|\Phi\|_{c b}=\left\|V_{1}^{*} V_{1}+V_{2}^{*} V_{2}\right\|$. This completes the proof.

COROLlaRY 4.12. Let $\delta$ be a nondegenerate coaction of a locally compact group $G$ on a $C^{*}$-algebra $A$, and let $W$ be a corepresentation of $G$ on a Hilbert space $\mathscr{H}$. If $\Phi: A \rightarrow \mathscr{B}(\mathscr{H})$ is a completely bounded linear operator satisfying

$$
\Phi \otimes i(\delta(a))=W(\Phi(a) \otimes 1) W^{*}
$$

for all $a \in A$, then there is a completely bounded operator $\phi: A \rtimes_{\delta} G \rightarrow$ $\mathscr{B}(\mathscr{H})$ such that, for all $a \in A$ and $f, g \in A(G)$,

$$
\phi\left(\left(1 \otimes M_{g}\right) \delta(a)\left(1 \otimes M_{f}\right)\right)=S_{g}(W) \Phi(a) S_{f}(W) .
$$

Proof. Choose $\pi, Y, T, V$ as in Theorem 4.11 and define

$$
\phi(z)=T(\pi \times Y)(z) V \quad \text { for } z \in A \rtimes_{\delta} G .
$$

Then $\phi$ is completely bounded with $\|\phi\|_{c b} \leq\|T\|\|V\|=\|\Phi\|_{c b}$. If $\bar{\phi}$ is the unique strictly continuous extension of $\phi$ to $M\left(A \rtimes_{\delta} G\right)$, then $\bar{\phi} \circ \delta(a)=T(\pi \times Y(\delta(a))) V=T \pi(a) V=\delta(a)$. Thus

$$
\|\Phi\|_{c b} \leq\|\bar{\phi}\|_{c b}\|\delta\|_{c b}=\|\bar{\phi}\|_{c b}=\|\phi\|_{c b} .
$$

From condition (c) of Theorem 4.11 we obtain

$$
\begin{aligned}
\phi\left(\left(1 \otimes M_{g}\right) \delta(a)\left(1 \otimes M_{f}\right)\right)=T(\pi \times Y)\left(\left(1 \otimes M_{g}\right) \delta(a)\left(1 \otimes M_{f}\right)\right) V \\
\quad=T S_{g}(Y) \pi(a) S_{f}(Y) V=S_{g}((T \otimes 1) Y) \pi(a) S_{f}(Y(V \otimes 1)) \\
\quad=S_{g}(W) T \pi(a) V S_{f}(W)=S_{g}(W) \Phi(a) S_{f}(W),
\end{aligned}
$$

as required. 
Corollary 4.13. Let $\delta$ and $\varepsilon$ be non-degenerate coactions of a locally compact group $G$ on $C^{*}$-algebras $A$ and $B$. If $\Phi: A \rightarrow B$ is a completely bounded linear operator such that $\Phi \otimes i(\delta(a))=\varepsilon(\Phi(a))$ for all $a \in A$, then there is a completely bounded linear operator $\Phi \times i: A \rtimes_{\delta} G \rightarrow B \rtimes_{\varepsilon} G$ satisfying

$$
\Phi \times i\left(\left(1 \otimes M_{g}\right) \delta(a)\left(1 \otimes M_{f}\right)\right)=\left(1 \otimes M_{g}\right) \varepsilon(\Phi(a))\left(1 \otimes M_{f}\right)
$$

for all $a \in A$ and $f, g \in C_{0}(G)$.

Proof. We choose a faithful nondegenerate representation $\pi \times W$ of $B \rtimes_{\varepsilon} G$ on a Hilbert space $\mathscr{H}$. Then

$$
\begin{aligned}
(\pi \circ \Phi) \times i(\delta(a)) & =(\pi \otimes i) \circ(\Phi \otimes i)(\delta(a)) \\
& =\pi \otimes i(\varepsilon(\Phi(a)))=W(\pi \circ \Phi(a) \otimes 1) W^{*}
\end{aligned}
$$

so that $\pi \circ \Phi$ is $\delta-\operatorname{Ad} W$ equivariant. By the previous corollary there is a completely bounded linear operator $\psi: A \rtimes_{\delta} G \rightarrow \mathscr{B}(\mathscr{H})$ such that

$$
\begin{gathered}
\psi\left(\left(1 \otimes M_{g}\right) \delta(a)\left(1 \otimes M_{f}\right)\right)=S_{g}(W)(\pi \circ \Phi)(a) S_{f}(W) \\
\quad=\pi \times W\left(\left(1 \otimes M_{g}\right) \varepsilon(\Phi(a))\left(1 \otimes M_{f}\right)\right)
\end{gathered}
$$

for all $f \in A(G)$; hence the outside equality is true for all $f \in C_{0}(G)$. We define $\Phi \times i=(\pi \times W)^{-1} \circ \psi$, which has the required properties.

5. Lifting equivariant maps twice. Let $\Phi:(A, G, \alpha) \rightarrow(B, G, \beta)$ be an equivariant completely bounded map. In $\S 3$, we constructed a completely bounded map $\Phi \times i: A \rtimes_{\alpha} G \rightarrow B \rtimes_{\beta} G$ which is equivariant for the dual coactions $\hat{\alpha}, \hat{\beta}$ of $G$. By Corollary 4.13 there is therefore a completely bounded map

$$
(\Phi \times i) \times i:\left(A \rtimes_{\alpha} G\right) \rtimes_{\hat{\alpha}} G \rightarrow\left(B \rtimes_{\beta} G\right) \rtimes_{\hat{\beta}} G
$$

satisfying

$$
(\Phi \times i) \times i\left(\hat{\alpha}\left(i_{A}(a) i_{G}(z)\right)\left(1 \otimes M_{f}\right)\right)=\hat{\beta}\left(i_{B}(\Phi(a)) i_{G}(z)\right)\left(1 \otimes M_{f}\right)
$$

for $a \in A, z \in C_{c}(G)$ and $f \in C_{0}(G)$. We now prove that the existence of this second lifting characterizes the complete boundedness of an equivariant map.

THEOREM 5.1. Let $\alpha$ and $\beta$ be actions of an infinite amenable locally compact group $G$ on $C^{*}$-algebras $A$ and $B$, respectively, and let $\Phi: A \rightarrow$ $B$ be a bounded operator satisfying $\Phi\left(\alpha_{s}(a)\right)=\beta_{s}(\Phi(a))$ for $a \in A$, 
$s \in G$. If there is a continuous linear map $\Theta$ from $\left(A \rtimes_{\alpha} G\right) \rtimes_{\hat{\alpha}} G$ to $\left(B \rtimes_{\beta} G\right) \rtimes_{\hat{\beta}} G$ satisfying

$$
\Theta\left(\hat{\alpha}\left(i_{A}(a) i_{G}(z)\right)\left(1 \otimes M_{f}\right)\right)=\hat{\beta}\left(i_{B}(\Phi(a)) i_{G}(z)\right)\left(1 \otimes M_{f}\right)
$$

for $a \in A, z \in C_{c}(G), f \in C_{0}(G)$, then $\Phi$ is completely bounded.

We would like to prove this theorem by showing that the composition of $\Theta$ with the duality isomorphisms $(A \rtimes G) \rtimes G \cong A \otimes \mathscr{K}$, $(B \rtimes G) \rtimes G \cong B \otimes \mathscr{K}$ of Imai and Takai ([10], see also [14, §6]) agrees with $\Phi \otimes i$ on the algebraic tensor product $A \odot \mathscr{K}$; this would imply that $\Phi \otimes i$ is bounded on $A \odot \mathscr{K}$, and hence that $\Phi$ is completely bounded. Unfortunately, we only know what $\Theta$ does on a set of generators which are not carried into $A \odot \mathscr{K}$ under the duality isomorphism. We get around this problem by using the following characterization of completely bounded maps, which may be of some independent interest.

Lemma 5.2. Let $A$ and $B$ be $C^{*}$-algebras, and let $\Phi: A \rightarrow B$ and $\Psi: A \otimes \mathscr{K} \rightarrow B \otimes \mathscr{K}$ be bounded linear maps. Then the following statements are equivalent:

(1) $\Phi$ is completely bounded and $\Psi=\Phi \otimes i$;

(2) $R S_{g} \circ \Psi=\Phi \circ R S_{g}$ for all $g \in \mathscr{K}^{*}$;

(3) $L S_{f} \circ \Psi=L S_{\text {f०Ф }}$ for all $f \in B^{*}$.

Proof. That (1) implies (3) follows from the continuity of both sides and a computation on elementary tensors. Now suppose (3) holds, and $g \in \mathscr{K}^{*}$. Then for any $f \in B^{*}$ and $y \in A \otimes \mathscr{K}$, the Fubini theorem gives

$$
\begin{aligned}
& f\left(R S_{g}(\Psi(y))\right)=g\left(L S_{f}(\Psi(y))\right) \\
& \quad=g\left(L S_{f \circ \Phi}(y)\right)=f \circ \Phi\left(R S_{g}(y)\right),
\end{aligned}
$$

which implies (2). Finally, suppose (2) holds. Then for $f \in B^{*}$, $g \in \mathscr{K}^{*}$ and $y \in A \odot \mathscr{K}$, we have

$$
\begin{array}{r}
f \otimes g(\Psi(y))=f\left(R S_{g} \circ \Psi(y)\right)=f\left(\Phi \circ R S_{g}(y)\right) \\
=((f \circ \Phi) \otimes g)(y)=(f \otimes g) \circ(\Phi \otimes i)(y) .
\end{array}
$$

Hence $\Phi \otimes i$ is bounded on $A \odot \mathscr{K}$, and its extension to $A \otimes \mathscr{K}$ agrees with $\Psi$. Since we can embed each $A \otimes M_{n}$ in $A \odot \mathscr{K}$ in such a way that $\Phi_{n}$ is the restriction of $\Phi \otimes i$, this shows that for each $n,\left\|\Phi_{n}\right\| \leq$ $\|\Phi \otimes i\|=\|\Psi\|$. This gives (1). 
Proof of Theorem 5.1. The duality isomorphism $\Theta$ is usually given in three stages:

$$
\begin{aligned}
& \left(A \rtimes_{\alpha} G\right) \rtimes_{\hat{\alpha}} G \stackrel{\phi}{\rightarrow}\left(A \otimes C_{0}(G)\right) \rtimes_{\alpha \otimes \tau} G \\
& \quad \stackrel{\psi}{\rightarrow}\left(A \otimes C_{0}(G)\right) \rtimes_{i \otimes \tau} G \stackrel{\theta}{\rightarrow} A \otimes \mathscr{K}\left(L^{2}(G)\right) ;
\end{aligned}
$$

we shall use the isomorphisms $\phi, \psi$ described in $[14,6.1,6.2]$ (and denoted there by $\Phi, \bar{\Psi})$. We fix a faithful representation $\pi$ of $A$ on $\mathscr{H}$, so that $A \rtimes_{\alpha} G$ is represented concretely on $\mathscr{H} \otimes L^{2}(G)$ via Ind $\pi=\tilde{\pi} \times \lambda$ [21, 7.7.5], and $\left(A \rtimes_{\alpha} G\right) \rtimes_{\hat{\alpha}} G$ acts on $\mathscr{H} \otimes L^{2}(G) \otimes L^{2}(G)$. If we similarly use a representation $\pi_{1}$ of $B$ on $\mathscr{H}_{1}$ to realize $(B \rtimes G) \rtimes G$ on $\mathscr{H}_{1} \otimes L^{2}(G) \otimes L^{2}(G)$, then for $a \in A, z \in C_{c}(G)$ and $f \in C_{0}(G)$ we have

$$
\begin{aligned}
\Theta(\hat{\alpha}(\tilde{\pi}(a) & \left.(1 \otimes \lambda(z)))\left(1 \otimes M_{f}\right)\right) \\
& =\hat{\beta}\left(\tilde{\pi}_{1}(\Phi(a))(1 \otimes \lambda(z))\right)\left(1 \otimes M_{f}\right) .
\end{aligned}
$$

We shall first compute $\psi \circ \phi$ on the generator

$$
c=\hat{\alpha}(\tilde{\pi}(a)(1 \otimes \lambda(z)))\left(1 \otimes M_{f}\right),
$$

and use this and the analogous computation for $(B \rtimes G) \rtimes G$ to pull $\Theta$ over to a map $\Psi_{1}=(\psi \circ \phi) \circ \Theta \circ(\psi \circ \phi)^{-1}$ of $\left(A \otimes C_{0}(G)\right) \rtimes_{i \otimes \tau} G$ into $\left(B \otimes C_{0}(G)\right) \rtimes_{i \otimes \tau} G$. We then rewrite property (3) of Lemma 5.2 in terms of the corresponding map $\Psi_{1}$ on $\left(A \otimes C_{0}(G)\right) \rtimes_{i \otimes \tau} G$, and finally verify that our $\Psi_{1}$ has the required property.

We shall view both crossed products $\left(A \otimes C_{0}(G)\right) \rtimes_{\alpha \otimes \tau} G$ and $\left(A \otimes C_{0}(G)\right) \rtimes_{i \otimes \tau} G$ as algebras on $\left(\mathscr{H} \otimes L^{2}(G)\right) \otimes L^{2}(G)$ via the faithful representations $(\pi \otimes M)^{\sim} \times \lambda$. From the proof of [14, Lemma 6.1] we have

$$
\begin{aligned}
\phi(c) & =\phi(\hat{\alpha}(\tilde{\pi}(a))) \phi(\hat{\alpha}(1 \otimes \lambda(z))) \phi\left(1 \otimes M_{f}\right) \\
& =(\pi \otimes M)^{\sim}(a \otimes 1)(1 \otimes 1 \otimes \lambda(z))(\pi \otimes M)^{\sim}(1 \otimes f) .
\end{aligned}
$$

By definition, $\psi$ is induced from the automorphism $\gamma$ of $A \otimes C_{0}(G)$ given by $\gamma(y)(s)=\alpha_{s}^{-1}(y(s))$ (see [14, Lemma 6.2]), which is the identity on $1 \otimes C_{0}(G)$. Hence

$$
\begin{aligned}
\psi \circ \phi(c) & =\psi\left((\pi \otimes M)^{\sim}(a \otimes 1)\right)(1 \otimes 1 \otimes \lambda(z)) \psi\left((\pi \otimes M)^{\sim}(1 \otimes f)\right) \\
& =(\pi \otimes M)^{\sim}(\gamma(a \otimes 1))(1 \otimes 1 \otimes \lambda(z))(\pi \otimes M)^{\sim}(1 \otimes f) .
\end{aligned}
$$

We now compute this operator on $L^{2}\left(G, \mathscr{H}_{\pi \otimes M}\right)=L^{2}\left(G, L^{2}(G, \mathscr{H})\right)$. First of all, for $g \in C_{0}(G, A)$ we have

$$
\begin{gathered}
{\left[(\pi \otimes M)^{\sim}(g)(\xi)(r)\right](t)=\left[(\pi \otimes M)\left(i \otimes \tau_{r}^{-1}(g)\right)(\xi(r))\right](t)} \\
=\pi\left(i \otimes \tau_{r}^{-1}(g)(t)\right)(\xi(r)(t))=\pi(g(r t))(\xi(r)(t)) .
\end{gathered}
$$


Therefore

$$
\begin{aligned}
{[\psi \circ \phi(c) \xi(r)](t) } & \\
& =\pi(\gamma(a \otimes 1)(r t))\left((1 \otimes 1 \otimes \lambda(z))(\pi \otimes M)^{\sim}(1 \otimes f)(\xi)(r)(t)\right) \\
& =\pi\left(\alpha_{t^{-1} r^{-1}}(a)\right)\left[\left(\int_{G} z(s)(\pi \otimes M)^{\sim}(1 \otimes f)(\xi)\left(s^{-1} r\right) d s\right)(t)\right] \\
& =\int_{G} z(s) f\left(s^{-1} r t\right) \pi\left(\alpha_{t^{-1} r^{-1}}(a)\right)\left(\xi\left(s^{-1} r\right)(t)\right) d s .
\end{aligned}
$$

If we write

$$
w(s)(u)=w_{z, f, a}(s)(u)=z(s) f\left(s^{-1} u\right) \alpha_{u^{-1}}(a),
$$

then $w \in C_{c}\left(G, C_{0}(G, A)\right)$, and a calculation shows that

$$
\left[(\pi \otimes M)^{\sim} \times \lambda(w)(\xi)(r)\right]=[\psi \circ \phi(c)(\xi)(r)](t) .
$$

Thus $\psi \circ \phi(c)$ is given by the continuous function $w_{z, f, a}$ defined by (2). Of course, equation (1) and exactly the same calculation show that

$$
\psi \circ \phi(\Theta(c))=w_{z, f, \Phi(a)} \in C_{c}\left(G, C_{0}(G, B)\right),
$$

which implies that

$$
\Psi_{1}\left(w_{z, f, a}\right)=(\psi \circ \phi) \circ \Theta \circ(\psi \circ \phi)^{-1}\left(w_{z, f, a}\right)=w_{z, f, \Phi(a)} .
$$

Notice that the usual approximation arguments show that the $w$ 's span a subspace of $C_{c}\left(G, C_{0}(G, A)\right)$ which is dense in the inductive limit topology, and hence is also dense in $C_{0}(G, A) \rtimes G$.

The isomorphism $\theta$ of $\left(A \otimes C_{0}(G)\right) \rtimes_{i \otimes \tau} G$ onto $A \otimes \mathscr{K}$ carries the generator

$$
i_{A \otimes C_{0}(G)}(a \otimes f) i_{G}(z)=(\pi \otimes M)^{\sim}(a \otimes f)(1 \otimes 1 \otimes \lambda(z))
$$

into the generator $a \otimes\left(M_{f} \lambda(z)\right)$ for $A \otimes \mathscr{K}\left(L^{2}(G)\right)$. Given $g \in A^{*}$, the slice map $S_{g}: A \otimes C_{0}(G) \rightarrow C_{0}(G)$ is completely bounded and $i \otimes \tau-\tau$ equivariant. Thus by Theorem 3.5 there is a continuous linear map $S_{g} \times i$ of $\left(A \otimes C_{0}(G)\right) \rtimes_{i \otimes \tau} G$ into $C_{0}(G) \rtimes_{\tau} G$ satisfying

$$
S_{g} \times i\left(i_{A \otimes C_{0}(G)}(a \otimes f) i_{G}(z)\right)=i_{C_{0}(G)}(g(a) f) i_{G}(z)
$$

and

$$
S_{g} \times i(w)(s)=S_{g}(w(s)) \quad \text { for } w \in C_{c}\left(G, C_{0}(G, A)\right) .
$$


The analogue of (4) for the algebra $B$ implies that for $g \in B^{*}$ we have a commutative diagram

$$
\begin{array}{ccc}
\left(B \otimes C_{0}(G)\right) \rtimes_{i \otimes \tau} G \stackrel{\theta}{\longrightarrow} B \otimes \mathscr{K}\left(L^{2}(G)\right) \\
S_{g} \times i \downarrow & S_{g} \downarrow \\
C_{0}(G) \rtimes_{\tau} G \quad \stackrel{M \times \lambda}{\longrightarrow} \quad \mathscr{K}\left(L^{2}(G)\right) .
\end{array}
$$

Thus for $w \in\left(A \otimes C_{0}(G)\right) \rtimes G$ we have

$$
M \times \lambda\left(S_{g} \times i\left(\Psi_{1}(w)\right)\right)=S_{g}\left(\theta\left(\Psi_{1}(w)\right)\right)=S_{g}(\Psi(\theta(w))) .
$$

Hence condition (3) of Lemma 5.2 is equivalent to

(6) $S_{g} \times i\left(\Psi_{1}(w)\right)=S_{g \circ \Phi} \times i(w)$ for $g \in B^{*}, w \in\left(A \otimes C_{0}(G)\right) \rtimes G$.

The slice map $S_{g}$ sends a continuous function $y \in C_{0}(G, B)$ to the function $s \rightarrow g(y(s))$ in $C_{0}(G)$, so condition (5) can be rewritten

$$
\left(\left(S_{g} \times i\right)(w)(s)\right)(u)=g(w(s)(u)) \text { for } w \in C_{c}\left(G, C_{0}(G, A)\right) .
$$

We can now compute:

$$
\begin{aligned}
S_{g} \times & i\left(\Psi_{1}\left(w_{z, f, a}\right)\right)(s)(u)=S_{g} \times i\left(w_{z, f, \Phi(a)}\right)(s)(u) \quad(\text { by }(3)) \\
& =g\left(w_{z, f, \Phi(a)}(s)(u)\right)=z(s) f\left(s^{-1} u\right) g\left(\beta_{u}^{-1}(\Phi(a))\right) \\
& =z(s) f\left(s^{-1} u\right) g\left(\Phi\left(\alpha_{u}^{-1}(a)\right)\right)=S_{g \circ \Phi} \times i\left(w_{z, f, a}\right)(s)(u) .
\end{aligned}
$$

Thus (6) holds for $w$ 's of the form $w_{z, f, a}$. Since such $w$ 's span a dense subspace of $\left(A \otimes C_{0}\right) \rtimes G$, and both sides of (6) are continuous, (6) holds. Thus $\Phi$ satisfies condition (3) of Lemma 5.2, and $\Phi$ is completely bounded. This completes the proof of Theorem 5.1.

There is an analogue of this result for completely bounded maps $\phi: A \rightarrow B$ which are equivariant for coactions $\delta, \varepsilon$ of $G$ on $A, B$. This time we first apply Corollary 4.13, and then use Theorem 4.5 to give a bounded map $(\phi \times i) \times i$ from $\left(A \rtimes_{\delta} G\right) \rtimes_{\hat{\delta}} G$ to $\left(B \rtimes_{\varepsilon} G\right) \rtimes_{\hat{\varepsilon}} G$. Again, the existence of such an extension implies that $\phi$ is completely bounded; the proof of this is similar to that of Theorem 5.1, except that it uses Katayama's duality theorem ([12], see also [14, $\S 4])$ in place of Imai and Takai's theorem. It turns out to be quite straightforward, so we shall omit the details.

TheOREM 5.3. Let $\delta, \varepsilon$ be coactions of an infinite amenable locally compact group $G$ on $C^{*}$-algebras $A, B$ respectively, and let $\Phi: A \rightarrow B$ be a continuous linear map satisfying

$$
\Phi\left(S_{f}(\delta(a))\right)=S_{f}(\varepsilon(\Phi(a))) \quad \text { for } a \in A, f \in A(G) .
$$


Suppose there is a continuous linear map $\Psi$ of $\left(A \rtimes_{\delta} G\right) \rtimes_{\hat{\delta}} G$ into $\left(B \rtimes_{\varepsilon} G\right) \rtimes_{\hat{\varepsilon}} G$ satisfying

$$
\Psi\left(i_{A \rtimes G}\left(\delta(a)\left(1 \otimes M_{f}\right)\right) i_{G}(z)\right)=i_{B \rtimes G}\left(\varepsilon(\Phi(a))\left(1 \otimes M_{f}\right)\right) i_{G}(z)
$$

for $a \in A, f \in C_{c}(G), z \in C_{c}(G)$. Then $\Phi$ is completely bounded.

6. The von Neumann case and multilinear operators. In this section we shall briefly outline and discuss normal liftings of completely bounded operators between von Neumann algebras and some results on multilinear operators. The situation for normal completely bounded linear operators between von Neumann algebras is simpler than the $C^{*}$-algebra situation, because there is a nice fixed point description of the cross product in terms of suitable actions on a von Neumann algebra tensor product [15]. We illustrate this idea by giving a normal operator-von Neumann algebra cross product version of the main action result (Theorem 3.5); there are similar coaction results but we do not discuss them.

THEOREM 6.1. Let $\alpha$ and $\beta$ be normal actions of a locally compact group $G$ on von Neumann algebras $M$ and $N$, respectively. If $\Phi$ is a completely bounded normal operator from $M$ into $N$ such that $\Phi \circ \alpha_{t}=$ $\beta_{t} \circ \Phi$ for all $t \in G$, and if $\Psi: v N(G) \rightarrow v N(G)$ is a normal completely bounded operator such that $\Psi \circ \lambda_{t}=\lambda_{t} \circ \Psi$ for all $t \in G$, then there is a natural completely bounded linear operator $\Phi \times \Psi: M \bar{\rtimes}_{\alpha} G \rightarrow N \bar{\rtimes}_{\beta} G$.

Proof. The operator $\Phi \otimes \Psi$ may be extended by complete boundedness and normality to a completely bounded normal operator from $M \bar{\otimes} \mathscr{B}\left(L^{2}(G)\right)$ into $N \bar{\otimes} \mathscr{B}\left(L^{2}(G)\right)$ : see [5, Lemma 1.5] or use the representation theorem for a completely bounded normal operator [19]. By $[15$, p. 23] it is sufficient to show that

$$
\tilde{\beta}_{t} \circ(\Phi \otimes \Psi)=(\Phi \otimes \Psi) \circ \tilde{\alpha}_{t}
$$

for all $t \in G$, because $\Phi \otimes \Psi$ will then map the $G$-invariant subalgebra of $M \bar{\otimes} \mathscr{B}\left(L^{2}(G)\right)$ into the $G$-invariant subalgebra of $N \bar{\otimes} \mathscr{B}\left(L^{2}(G)\right)$, and these algebras are naturally isomorphic to $M \bar{\rtimes}_{\alpha} G$ and $N \bar{\rtimes}_{\beta} G$, respectively. By definition $\tilde{\alpha}_{t}=\alpha_{t} \otimes \lambda_{t}$ and $\tilde{\beta}_{t}=\beta_{t} \otimes \lambda_{t}$ so that (1) becomes $\beta_{t} \circ \Phi \otimes \lambda_{t} \circ \Psi=\Phi \circ \alpha_{t} \otimes \Psi \circ \lambda_{t}$, which holds by hypothesis.

There are multilinear versions of the results in this paper based on the definition of a completely bounded multilinear operator in [4] and 
[20]. We shall state two results of this type for actions of a locally compact group on a $C^{*}$-algebra, and indicate how the proofs may be obtained by combining the techniques of $\S 3$ and ([4] or [20]).

Proposition 6.2. Let $\alpha$ be an action of a locally compact amenable group $G$ on a $C^{*}$-algebra $A$, and let $g \mapsto u_{g}$ be a unitary representation of $G$ on a Hilbert space $\mathscr{H}$. Suppose that $\Phi$ is a completely bounded $n$-linear operator from $A^{n}$ into $\mathscr{B}(\mathscr{H})$. If $\Phi$ is equivariant, in the sense that $\Phi\left(\alpha_{t}\left(x_{1}\right), \ldots, \alpha_{t}\left(x_{n}\right)\right)=u_{t} \Phi\left(x_{1}, \ldots, x_{n}\right) u_{t}^{*}$, then there are

(1) covariant representations $\left(\mathscr{H}_{j}, \pi_{j}, W_{j}\right)$ of $(A, G, \alpha)$ for $1 \leq j \leq n$,

(2) continuous linear "bridging" operators

$$
\mathscr{H} \stackrel{V_{n}}{\rightarrow} \mathscr{H}_{n} \stackrel{V_{n-1}}{\rightarrow} \mathscr{H}_{n-1} \stackrel{V_{n-2}}{\rightarrow} \ldots \stackrel{V_{n}}{\rightarrow} \mathscr{H}_{1} \stackrel{V_{0}}{\rightarrow} \mathscr{H}
$$

such that

(a) $\Phi\left(a_{1}, \ldots, a_{n}\right)=V_{0} \pi_{1}\left(a_{1}\right) V_{1} \cdots V_{n-1} \pi_{n}\left(a_{n}\right) V_{n}$,

(b) $u_{t} V_{0}=V_{0} W_{1}(t), W_{j}(t) V_{j}=V_{j} W_{j+1}(t)(1 \leq j \leq n-1)$, and $W_{n}(t) V_{n}=V_{n} u_{t}$ for all $t \in G$,

(c) $\|\Phi\|_{c b}=\left\|V_{0}\right\| \cdots\left\|V_{n}\right\|$.

Outline of the proof. The basic proof in [4] and [20] is by reduction to the symmetric case using a standard $2 \times 2$ matrix symmetrization $[4,5.3]$ followed by induction on $n$ with step length $2[4,4.1]$. The induction ends with $n=1$, which was dealt with in $\S 3$, or $n=0$, which is just a fixed intertwining operator on a Hilbert space (note, $n$ here is $k$ in [4]). The induction starts with the existence of a completely positive linear operator $\psi: A \rightarrow \mathscr{B}(\mathscr{H})$ such that $\|\psi\| \leq\|\Phi\|_{c b}$ and

$$
\begin{aligned}
-\psi_{k}\left(x^{*} x\right)\left\|a_{2}\right\| \cdots\left\|a_{n-1}\right\| & \leq \Phi_{k}\left(x^{*}, a_{2}, \ldots, a_{n-1}, x\right) \\
& \leq \psi_{k}\left(x^{*} x\right)\left\|a_{2}\right\| \cdots\left\|a_{n-1}\right\|
\end{aligned}
$$

for all $x, a_{2}, \ldots, a_{n-1} \in M_{k}(A)$ with $\left(a_{2}, \ldots, a_{n-1}\right)=\left(a_{n-1}^{*}, \ldots, a_{2}^{*}\right)$ [4, Theorem 2.8]. The completely positive linear operator $\psi$ is now averaged by the invariant mean $m$ of $G$ to obtain a completely positive $G$-invariant linear operator $\phi$ defined by

$$
\langle\phi(x) \xi, \eta\rangle=m\left(t \mapsto\left\langle u_{t}^{*} \psi\left(\alpha_{t}(x)\right) u_{t} \xi, \eta\right\rangle\right)
$$

$(x \in A ; \xi, \eta \in \mathscr{H})$. In inequality $(*)$ we replace $a_{j}$ by $\alpha_{t, k}\left(a_{j}\right)$ where $\alpha_{t, k}=\alpha_{t} \otimes I_{k}$ is the $k$-fold amplification of $\alpha$, we replace $x$ by $\alpha_{t, k}(x)$, and act on the resulting inequality by $\operatorname{Ad}\left(u_{t}^{*} \otimes I\right)$. The $G$-invariance of $\Phi$ implies that $\Phi_{k}$ is invariant under the $k$-fold amplification of the actions of $G$, so a similar inequality results with $\psi_{k}\left(x^{*} x\right)$ replaced by

$$
\left(\operatorname{Ad}\left(u_{t}^{*}\right) \circ \psi \circ \alpha_{t}\right)_{k}\left(x^{*} x\right) \text {. }
$$


Averaging this by the invariant mean of $G$ yields inequality $(*)$ with $\psi$ replaced by the completely positive $G$-invariant linear operator $\phi$. We now combine the $G$-invariant Stinespring Theorem (Lemma 3.1) with [4, Lemma 3.1]. Together these give rise to a covariant representation of $(A, G, \alpha)$ on a Hilbert space $\mathscr{K}$ and a completely bounded $G$-invariant symmetric $(n-2)$ linear operator $\Psi$ from $A^{n-2}$ into $\mathscr{B}(\mathscr{K})$ such that $\Phi\left(a_{1}, \ldots, a_{n}\right)=V^{*} \pi\left(a_{1}\right) \Psi\left(a_{2}, \ldots, a_{n-1}\right) \pi\left(a_{n}\right) V$, for all $a_{1}, \ldots, a_{n} \in A$; where $\pi$ and $V$ are the representation of $A$ and the linear operator from $\mathscr{H}$ into $\mathscr{K}$ given by Lemma 3.1 (note $\pi=\theta$ of [4, Lemma 3.1]). This construction has reduced the problem from $n$ to $(n-2)$ with the same hypothesis, and completes the sketch.

Proposition 6.3. Let $\alpha$ and $\beta$ be actions of an amenable group $G$ on $C^{*}$-algebras $A$ and $B$, respectively. If $\Phi$ is a completely bounded $n$-linear operator from $A^{n}$ into $B$, which is $G$-invariant in the sense that

$$
\beta_{t} \Phi\left(a_{1}, \ldots, a_{n}\right)=\Phi\left(\alpha_{t}\left(a_{1}\right), \ldots, \alpha_{t}\left(a_{n}\right)\right),
$$

then there is a completely bounded $n$-linear operator $\Phi \times i$ from $\left(A \rtimes_{\alpha} G\right)^{n}$ into $B \rtimes_{\beta} G$ such that

(1) $\|\Phi \times i\|_{c b}=\|\Phi\|_{c b}$, and

$$
\begin{aligned}
&(\Phi \times i)\left(y_{1}, \ldots, y_{n}\right)(t) \\
&=\iint \cdots \int \Phi\left(y_{1}\left(s_{1}\right), \alpha_{s_{1}}\left(y_{2}\left(s_{2}\right)\right), \alpha_{s_{1} s_{2}}\left(y_{3}\left(s_{3}\right)\right),\right. \\
&\left.\ldots, \alpha_{s_{1} s_{2} \cdots s_{n-1}}\left(y_{n}\left(s_{n-1}^{-1} \cdots s_{1}^{-1} t\right)\right)\right) d s_{1} \cdots d s_{n-1}
\end{aligned}
$$

for all $y_{1}, \ldots, y_{n} \in C_{c}(G, A)$ and $t \in G$.

This result is proved from Proposition 6.2 in the same way that Theorem 3.5 is deduced from Theorem 3.3. The other parts of Theorem 3.5 also carry over but we omit these. We also omit the corresponding multilinear versions of our results on coactions.

REMARK 6.4. The above multilinear results may be applied in the study of equivariant completely bounded cohomology. There is a discussion of completely bounded cohomology for $C^{*}$-subalgebras of $\mathscr{B}(\mathscr{H})$ in [3]. Here we shall restrict attention to a simple special case.

Suppose that $A$ is a subalgebra of another $C^{*}$-algebra $B$, and $\alpha: G \rightarrow$ $\operatorname{Aut}(B)$ is an action of an amenable group $G$, which restricts to an action of $G$ on $A$. We let $C_{a b}^{n}(A, B ; G)$ denote the space of equivariant completely bounded $n$-linear maps $\Phi: A^{n} \rightarrow B$. Observe that the 
usual Hochschild coboundary operator $\delta$ maps equivariant maps to equivariant maps. Now define the equivariant completely bounded Hochschild cohomology groups to be those of the complex

$$
B^{G} \stackrel{\delta}{\rightarrow} C_{c b}^{1}(A, B ; G) \stackrel{\delta}{\rightarrow} C_{c b}^{2}(A, B ; G) \stackrel{\delta}{\rightarrow} \cdots
$$

Denote these groups by $H_{c b}^{n}(A, B ; G)$. For $n \geq 1$ we define operators

$$
j_{G}: C_{c b}^{n}(A, B ; G) \rightarrow C_{c b}^{n}\left(A \rtimes_{\alpha} G, B \rtimes_{\alpha} G\right)
$$

by $j_{G}(\Phi)=\Phi \times i$, and for $n=0$ we define $j_{G}=i_{G}: B^{G} \rightarrow M\left(B \rtimes_{\alpha} G\right)$. Then a calculation using property (2) of Proposition 6.3 shows that $j_{G}$ commutes with $\delta$, so we get induced homomorphisms

$$
j_{G}^{*}: H_{c b}^{n}(A, B ; G) \rightarrow H_{c b}^{*}(A \rtimes G, B \rtimes G) .
$$

Thus our construction induces a homomorphism from equivariant completely bounded cohomology into the completely bounded cohomology of the crossed product. This may play a useful rôle in studying equivariant cohomology.

\section{REFERENCES}

[1] W. B. Arveson, Subalgebras of $C^{*}$-algebras, Acta Math., 123 (1969), 141-224.

[2] R. C. Busby, Double centralizers and extensions of $C^{*}$-algebras, Trans. Amer. Math. Soc., 132 (1968), 79-99.

[3] E. Christensen, E. G. Effros, and A. M. Sinclair, Completely bounded multilinear maps and $C^{*}$-algebraic cohomology, Inventiones Math.

[4] E. Christensen and A. M. Sinclair, Representations of completely bounded multilinear operators, J. Funct. Anal., 72 (1987), 151-181.

[5] J. de Canniere and U. Haagerup, Multipliers of the Fourier algebras of some simple Lie groups and their discrete subgroups, Amer. J. Math., 107 (1985), 455-500.

[6] M. Enock and J.-M. Schwartz, Algèbres de Kac moyennables, Pacific J. Math., 125 (1986) 363-379.

[7] P. Eymard, L'algèbre de Fourier d'un groupe localement compact, Bull. Math. Soc. France, 92 (1964), 181-236.

[8] E. Effros and Z-J. Ruan, On matricially normed spaces, preprint.

[9] E. E. Granirer, Weakly almost periodic and uniformly continuous functionals on the Fourier algebra of any locally compact group, Trans. Amer. Math. Soc., 189 (1974), 371-382.

[10] S. Imai and $\mathrm{H}$. Takai, On a duality for $C^{*}$-crossed products by a locally compact group, J. Math. Soc. Japan, 30 (1978), 495-504.

[11] G. G. Kasparov, Hilbert $C^{*}$-modules: Theorems of Stinespring and Voiculescu, J. Operator Theory, 4 (1980), 133-150.

[12] Y. Katayama, Takesaki's duality for a non-degenerate coaction, Math. Scand., 55 (1985), 141-151.

[13] M. B. Landstad, Duality for covariant systems, Trans. Amer. Math. Soc., 248 (1979), 223-267. 
[14] M. B. Landstad, J. Phillips, I. Raeburn, and C. E. Sutherland, Representations of crossed products by coactions and principal bundles, Trans. Amer. Math. Soc., 299 (1987), 747-784.

[15] Y. Nakagami and M. Takesaki, Duality for crossed products of von Neumann algebras, Lecture Notes in Math., Vol. 731, Springer Verlag, Berlin, 1979.

[16] W. L. Paschke, Inner product modules over $B^{*}$-algebras, Trans. Amer. Math. Soc., 182 (1973), 443-468.

[17] V. I. Paulsen, A covariant version of Ext, Michigan Math. J., 29 (1982), 131-142.

[18] Completely bounded maps on $C^{*}$-algebras and invariant operator ranges, Proc. Amer. Math. Soc., 86 (1982), 91-96.

[19] _ Completely Bounded Maps and Dilations, Pitman Press, Pitman Research Notes in Math., 146 (1986).

[20] V. I. Paulsen and C. Y. Suen, Commutant representations of completely bounded maps, J. Operator Theory, 13 (1985), 87-101.

[21] G. K. Pedersen, $C^{*}$-Algebras and their Automorphism Groups, Academic Press, London and New York, 1979.

[22] I. Raeburn, On crossed products and Takai duality, Proc. Edin. Math. Soc., 31 (1988), 321-330.

[23] W. F. Stinespring, Positive functions on $C^{*}$-algebras, Proc. Amer. Math. Soc., 6 (1955), 211-216.

[24] M. Takesaki, Theory of operator algebras, I, Springer Verlag, Berlin, 1979.

[25] G. Wittstock, Ein operatorwertiger Hahn-Banach Satz, J. Functional Anal., 40 (1981), 127-150.

[26] On Matrix Order and Convexity, Functional Analysis: Surveys and Recent Results, Math. Studies 90, North Holland, Amsterdam 1984, pp. 175-188.

Received June 15, 1987. This research was supported by the Australian Research Grants Scheme. The second author was also supported by the Royal Society, U.K. and the third author was also supported by the National Science Foundation, U.S.A.

UNIVERSity OF New South WALES

Kensington, N.S.W. 2033, Australia

UNIVERSITY OF EDINBURGH

EDINBURGH EH9 3JZ, SCOTLAND

AND

Dartmouth College

HANOVER, NH 03755, USA 